\title{
Metabolic energy expenditure and the regulation of movement economy
}

\author{
W. A. SPARROW \\ Deakin University, Victoria, Australia \\ and \\ K. M. NEWELL \\ Pennsylvania State University, University Park, Pennsylvania
}

\begin{abstract}
Over the years, various psychological theories have embraced notions of economy, efficiency, or least effort to explain how complex movement sequences are organized and modified. The purpose of the present paper was to synthesize various perspectives on this issue, to identify a common hypothesis, and to propose a conceptual framework that explains how movement economy is regulated. The framework presented here postulates that adaptive movement patterns emerge as a function of the organism's propensity to minimize metabolic energy expenditure with respect to task, environment, and organism constraints to action. An important role is also proposed for interoceptive sensory information in guiding motor skill learning and control. The paper concludes by suggesting future directions in four areas of movement economy research that contribute to understanding the learning and control of movement in both human and nonhuman organisms.
\end{abstract}

One of the most pervasive characteristics of the everyday performance of motor skills is the propensity to complete the task with the least energy expenditure. Economical movements are those that achieve the task goal with relatively low metabolic energy expenditure for the given task demands. Despite the common characterization of well-practiced movements as economical, only in recent years has research interest focused on understanding the relationship between metabolic energy expenditure and motor learning and control. The purpose of this paper is to examine the proposition that, from the repertoire of all possible movement sequences, humans and other organisms tend to adopt a coordination and control solution that is economical in terms of metabolic energy expenditure. We propose a conceptual framework for understanding how movement sequences emerge to accommodate the task and environment constraints that are imposed on an adaptive organism.

Motor skill researchers have traditionally focused on the goal-directed nature of skilled activities, with certainty and accuracy of goal attainment being the usual performance measures. It is important to note, however, that many formal definitions of skill have included terms such as efficiency or smoothly and efficiently to supplement the goal-directed description of skilled motor performance (see, e.g., Guthrie, 1935; Robb, 1972; Singer, 1968). De-

The authors thank Ross Day for valuable comments on the manuscript and David Williams for preparing the figures. K.M.N. is in the Department of Kinesiology at Penn State. Address correspondence to W. A. Sparrow, School of Human Movement, Deakin University, 221 Burwood Hwy., Burwood 3125, Australia (e-mail: sparrow@deakin. edu.au). spite such long-standing references to economy of movement, it has not traditionally been an important issue in motor skills research, possibly because tasks demanding little metabolic energy expenditure have predominantly been studied (see Newell, 1985; Sparrow, 1983). The lack of interest in metabolic energy expenditure was also sustained by the influential papers of Adams $(1968,1971)$ and Keele (1968). Adams's (1968) discussion of sensory feedback in the early learning experiments with rats focused almost exclusively on the potential contribution of proprioception and exteroception to the learning and control of movement in humans. Consequently, sensory information concerning metabolic energy expenditure has rarely been considered important for human motor behavior, even within closed-loop feedback theories of motor control. As we show in a later section, however, issues of effort and energy expenditure and minimum principles related to time, distance, and force were a major influence in early psychological theorizing about learning, but this tradition did not continue in the motor skills research of the 1940 s and beyond.

In an earlier review, Sparrow (1983) proposed that considerations of metabolic energy expenditure might provide insights into the organization of movement. Two possibilities were raised to account for the efficiency or smoothness of movement skill. The first was that these characteristics might emerge from a more fundamental organizing principle, so that metabolic energy expenditure per se is not regulated, but, rather, metabolic energy expenditure is minimized as a consequence of increased proficiency at a motor task. The second possibility was that "efficiency of performance may be viewed as a condition which specifies a priori a particular biokinematic organization of the 
organism" (p. 238). From this point of view, the propensity to minimize metabolic energy expenditure would be considered a primary mechanism for regulating the coordination and control of movement rather than a property that is a consequence of, or emerges from, some other organizing principle. In this paper, we provide further evidence to support the hypothesis that metabolic energy regulation is a fundamental principle underlying the learning and control of motor skills.

At the time of our first speculations on movement economy, little direct experimental work had been undertaken on such issues. Over the last 10-15 years, however, there has emerged a substantive literature about metabolic energy expenditure and movement economy. In this paper, we update the earlier review of this literature (Sparrow, 1983 ) and propose a constraints-based framework for understanding the relationship between metabolic energy expenditure and motor coordination and control. The framework outlined here considers economical movement sequences as emerging from the interaction of task, organism, and environment constraints (Newell, 1985, 1986) and is explicit about how metabolic energy is expended to accommodate these constraints. Our review is broad in scope, so that skills involving small muscle groups and/or relatively limited movement are also considered, because issues related to movement economy are not specific to high-energy-demanding tasks. For example, experiments showing measurable variation in metabolic rates have been performed with reaction time tasks (Sherwood, Allen, Obrist, \& Langer, 1986) and video games (Turner \& Carroll, 1985).

Much of the literature reviewed here concerns human motor behavior. Our thinking has, however, been influenced by early learning theorists, such as Tolman and Hull, and more recent operant research with rats (see, e.g., Brener, 1986) has also assisted in refining our perspective on human motor learning and control. The review has also been influenced by the work of contemporary theorists in movement coordination and control, who have utilized advances in theoretical biology to elucidate the role of metabolic energy in movement regulation (see, e.g., Kugler, Kelso, \& Turvey, 1980, 1982; Kugler $\&$ Turvey, 1987). Drawing on natural examples of physical order, such as the hexagonal patterns of honeycombs and the development of river meanders, they have suggested that minimization of energy or work is an organizing principle common to natural phenomena. It has been proposed that similar principles underlie the development of biological systems. In the development of motor coordination, for example, reactive forces become more effectively used, and it is possible that the muscle torque patterns that exploit reactive forces are also metabolically economical (Turvey \& Fitzpatrick, 1993). Consonant with this view, we hypothesize that processes of metabolic energy expenditure regulation explain both the short-term control of movement and the learning of adaptive motor behavior with practice. To explain how metabolic energy expenditure is regulated, the final sec- tions of the paper describe how sensory information concerning metabolic energy expenditure is available from both the task and the environment. In this discussion of the informational support for action, the role of sensory information in mediating action is reassessed. This discussion is particularly important, because the orthodoxy in motor behavior research has been that interoceptive information, the information from internal organs that provides some of the sensory stimulation concerning metabolic energy expenditure, "has questionable relevance for motor behavior" (Schmidt, 1982, p. 193). In contrast, we view motor behavior as, fundamentally, the process by which organisms convert chemical energy, through the metabolism of foodstuffs, to mechanical energy, in order to interact adaptively with their environment. Thus, the role of sensory information in signaling the economy of motor responses is considered central to the study of motor behavior. The present paper proceeds by first defining economy and metabolic energy expenditure. The following section discusses coordination and control of movement and the various constraints on action that influence the emergence of adaptive movements. The relationship between economy, coordination, control, and constraints is then presented formally by way of a conceptual framework. The empirical support for the framework is then put forward as four subdomains of economy research that provide evidence of economical adaptations to task and environment constraints. The subdomains are self-optimization, entrainment, (nonhuman) animal learning, and human learning. In presenting evidence to support the hypothesized relationship between metabolic energy expenditure and movement coordination and control, contemporary quantitative theories of motor control are also discussed. Although this literature constitutes a departure from the major focus on metabolic energy expenditure, it is too important to omit, given its emphasis on minimum principles relating to movement mechanics. The final sections of the paper critique the long-standing issue of the informational support for action, and we suggest how economy of metabolic energy expenditure is achieved. The paper's fundamental contribution to the discussion of the sensory control of movement is the proposal that sensory information from internal organs, interoceptive information, should be considered a major component of the sensory input by which movements are coordinated and controlled. We conclude the paper by suggesting future research directions in the subdomains described above.

\section{EFFICIENCY, METABOLIC ENERGY, AND ECONOMY DEFINED}

\section{Efficiency and Metabolic Energy}

The major focus of this paper is the question of how metabolic energy is expended in such a way that the economy of movement is maximized. Before defining economy, it is useful first to define the related term efficiency, because some of the studies reviewed are con- 
cerned with both efficiency and economy and it is easier to explain economy, having first defined efficiency. Efficiency, as understood by exercise physiologists and biomechanists, has a number of problems of definition that are too extensive to cover in detail here. The reader is advised to consult comprehensive reviews of movement efficiency, such as those of Cavanagh and Kram (1985a, $1985 \mathrm{~b}$ ), for a detailed exposition. For the present purpose, efficiency is defined as the ratio of mechanical work done to metabolic energy expended.

Efficiency, so defined, is often expressed as a percentage, according to the following formula:

$$
\text { Efficiency }(\%)=\frac{\text { Mechanical Work Done }}{\text { Energy Used for Work }} * 100 \text {. }
$$

The numerator of the efficiency equation will be examined first. In performing any motor activity, mechanical work is done in order to meet task demands. Task demands arise from the operation of machines, the use of implements such as tools, and in moving the limbs unencumbered by such devices, as in walking or swimming. One of the major difficulties in evaluating movement efficiency is measuring the mechanical work done in performing motor tasks. In laboratory settings, mechanical work is often measured with an ergometer, such as a bicycle fitted with a resistive device. With ergometers, work done can be calculated as a function of the force exerted and the distance over which the force is applied. Work per unit time is power, so that work rate, or power output in watts, is the unit in the numerator of the efficiency equation. When an ergometer is not used, the mechanical work done can be calculated by treating the body as a point-mass, so that work done is the product of body mass and distance traveled, divided by time. One problem with this calculation is that it is insensitive to variations in mechanical work that depend on how the limbs are moved. This restriction has led to the development of linked segment models (see, e.g., Aleshinsky, 1986; Winter, 1979), in which the body is modeled as a mechanical system of linked segments. With such models, work done can be calculated from the displacement, movement time, and mass of the individual segments. We refer later to an experiment by Sparrow and Irizarry-Lopez (1987) that used linked segment analyses in conjunction with metabolic energy cost measures to determine whether efficiency improves with practice because of refinements in limb movements.

The denominator of the efficiency equation is metabolic energy expenditure. Metabolic energy is derived from food, mainly fat and carbohydrates, that is converted to chemical energy, which, in turn, is converted to mechanical energy through muscular contraction. When energy is expended in muscular contraction, heat is produced, and the amount of heat produced by food metabolism is equivalent to the heat liberated by the body. In utilizing food as chemical energy to contract the muscles, oxygen is consumed, and the amount of oxygen consumed during exercise can be used as an indirect method for deter- mining heat production. In all the studies reviewed in this paper, metabolic energy expenditure is derived by indirect calorimetry, in which oxygen consumption is determined from samples of expired air. From oxygen consumption, the amount of heat produced can be determined on the basis of the type of foodstuffs (fat, carbohydrates, or protein) being utilized; this conversion provides the caloric equivalent of the oxygen consumed. To provide the same units of measurement as the numerator of the efficiency equation, the work equivalent of the oxygen consumed (in kilocalories) can be calculated, given that 1 kilocalorie ( 1,000 calories) of heat energy is equivalent to 426.8 newton-meters of work. Dividing Newton meters by time (in seconds) gives the number of watts provided by the metabolism of foodstuffs. The term metabolic energy, used throughout the paper is, therefore, energy in watts supplied by the metabolism of food; for brevity, the term energy alone is also used.

In summary, efficiency in the above equation represents the amount of metabolic energy that is converted into work to meet task demands, and the major focus of this review is how organisms regulate metabolic energy expenditure to perform work that meets motor task demands. There are, however, losses of metabolic energy to other functions, such as thermoregulation. For completeness, the constraints framework we have developed includes a component that represents the direct environment demands on metabolic energy expenditure associated with, for example, the ambient temperature and humidity.

\section{Economy}

In agreement with Cavanagh and Kram (1985a), we propose that, for understanding everyday motor skills, the concept of economy is more useful than is efficiency, because it is often impossible to accurately measure the mechanical work done. If a task is devised that imposes apparently fixed mechanical power demands-such as the power demands of the various ergometers used in respiratory physiology laboratories - changes in metabolic energy expenditure are usually interpreted as reflecting changes in efficiency. But, as individuals adapt to a motor task with practice, the internal mechanical work associated with coordinating and controlling the limbs may decrease over time. In such circumstances, the important performance criterion is metabolic energy expenditure, and both efficiency and economy may have improved. Most of the studies reviewed here concern movement economy, because they focus on everyday tasks in which the total mechanical work done cannot be accurately determined. For practical purposes, therefore, the primary consideration in the performance of motor tasks is the metabolic energy expended to achieve the objective or goal. Economy can, therefore, be defined as the metabolic energy expended to achieve the task goal. It was indicated above that heat energy can be calculated on the basis of the volume of oxygen consumed and the type of food metabolized. Many studies, however, do not 
report this conversion, because, for practical purposes, the variation in heat energy that is due to dietary considerations is small. Rather, metabolic energy expenditure is usually reported as oxygen consumption per unit of body mass. Unless indicated otherwise, the term economy will be used to make comparisons in terms of oxygen consumption per unit of body weight for performing a given task.

\section{COORDINATION, CONTROL, AND CONSTRAINTS ON MOVEMENT}

Adaptive movement patterns emerge as a function of the organism's propensity to conserve metabolic energy, on the one hand, and of the demands arising from constraints imposed by the organism, the task, and the environment, on the other. Constraints are the boundaries, limitations, or design features that apply restrictions to movement. The three categories of constraint that influence the pattern of limb coordination and control are considered below. To be specific about how the movement pattern is adapted to accommodate constraints, it is necessary to distinguish the terms coordination and control. These terms are important for our analysis, because the characteristic of movement that we define as coordination can change spontaneously to a new organization in response to a change in either task or environment constraints. The change in the patterns of movement coordination also appear to be related to principles of movement economy.

\section{Coordination and Control}

Some adaptations to the demands of motor tasks arise from the tuning, scaling, or parameterizing of an existing coordination mode, whereas others constitute a fundamental reorganization of the movement-what we would call a change in coordination. We draw upon Kugler et al. $(1980,1982)$ for our operational definitions of coordination, control, and the related term skill (Newell, 1985; Sparrow, 1992). Although coordinations can be described microscopically - in terms of configurations of tensile states or the patternings of cellular and vascular activities (Turvey, 1990) - for the present purpose, we will focus on coordinations described at the macroscopic level of limbs and limb segments. At this level, coordination can be defined either as the relationship between the movements of limb segments of the same limb-what we can refer to as intralimb coordination-or as the relationship between the movements of two different limbs (or the segments of different limbs), which can be referred to as interlimb coordination. Intralimb coordination therefore defines the topology of a single limb's movement-that is, the spatial relations of the limb segments over time - whereas interlimb coordination defines how two or more limbs maintain a temporal relationship to each other.

Control refers to the absolute magnitude of the limb or limb segment movement, expressed as a property of the kinetics or kinematics. For example, the amplitude, ve- locity, acceleration, or force of the movement would dictate the parameterization of the coordination function. If, for example, the arm was extended in order to reach and grasp an object, such as a glass, the relative motions (coordination) of the forearm and upper arm could remain unchanged while the speed or extent (control) of the reaching movement varied. The goal of a motor task usually incorporates an optimum or target value of variables such as amplitude or velocity, and, as in the case of reaching to pick up a glass, a well-controlled movement is one that satisfactorily approximates the optimum or target outcome. Thus, coordination and control are embedded concepts in the execution of a skill, and the overall goal of the task could be achieved by changing coordination, control, or both. Skill is an emergent property and can be defined operationally in terms of the ability to economically coordinate and control the movement to achieve the task goal.

\section{Organism Constraints}

In order to understand why a specific pattern of coordination and control is observed, it is important to consider the influence of the physical design, or constraints, of the organism itself. Organism constraints can be defined with respect to any perceptual, physical, or cognitive parameters and at any level of analysis, from behavioral to cellular. Organism constraints at the behavioral, or macro, level are those that impose physical limitations on the body's ability to perform mechanical work that meets task demands. Cavanagh and Kram (1985b) considered such constraints in a discussion of individual differences in movement economy. They gave the example of two individuals with the same body mass but with one having lighter limbs. When running at the same speed and, therefore, accommodating the same task constraints, the lighter limbed individual will perform less work and, other things being equal, expend less metabolic energy. Cavanagh and Kram (1985b) discussed other anatomical differences that influence movement economy, such as the point of muscle insertion relative to the joint centers. On the basis of mechanical principles, it is reasonable to assume that other characteristics of limb segment architecture, such as the distribution of mass within a segment and the relative length of limb segments, will influence the capacity to economically accommodate task constraints.

There are also organism constraints inherent in the qualities of the nervous system and in the properties of skeletal muscle, such as the rate at which muscles can generate force. Cavanagh and Kram (1985b) suggested, for example, that muscle fiber orientation and length can influence economy. Similarly, in a mechanical analysis of movement economy, Nelson (1983) discussed performance constraints. These are similar to our organism constraints and are defined as "magnitude limits on the forces and torques which can be generated, and on how rapidly they can be changed" (p. 135). In the short term, movement economy is, therefore, highly constrained by various anatomical and physiological characteristics of the organism. 
Organism constraints can be considered fixed in the short term, and the organism's adaptation to task and environment demands can be viewed as a motor control problem for the perceptual-motor system. Solutions to the short-term problem of adaptation are found in various preferred modes of coordination and control, such as a preferred rate or amplitude of limb movement. In some cases, the preferred solution to the motor control problem is realized in a fundamental reorganization of interlimb timing, such as the abrupt changes in relative coordination that is characteristic of the gait transitions in quadrupeds. There are, however, limitations to the organism's capacity to economically accommodate task and environment constraints through motor control strategies. In the long term, therefore, there are processes of adaptation that are associated with learning and development. As we show later in the paper, organisms learn to refine movements with practice, so that metabolic energy expenditure is reduced, even when the task constraints remain unchanged. Although, as yet, there has been little empirical work on the influence of physical growth on movement economy, it is reasonable to hypothesize that developmental changes in the architecture, or structure and form, of an organism also allow more economical long-term adaptation to task and environment constraints. Thus, the critical concept underlying an understanding of organism constraints is adaptation. In adapting motor patterns to meet task demands, constraints of the organism are accommodated rather than overcome, because all living organisms have fundamental constraints that limit adaptation to the environment in their performance of various perceptualmotor tasks.

\section{Environment Constraints}

Environment constraints are those constraints external to the organism that impose metabolic energy demands directly rather than through the task. Generally, the ambient environmental conditions are not manipulated by the experimenter, except when their influence is of specific interest, as when performing in extremes of temperature or humidity. Whipp and Wasserman (1969), for example, pointed out that environmental conditions influence the stress on an organ system, citing as an example the observation that cardiac output may be less when performing at low temperatures. Thus, studies of motor performance under environmental conditions of temperature, humidity, illumination, and noise would be instances of the systematic variation of environment constraints. Gravitational force is also an important environment constraint influencing the magnitudes and configurations of forces exerted on the organism. Although the Earth's gravitational constant acts as a global constraint on an organism's movements, the gravitational forces that must be overcome by specific patterns of muscle contraction change continuously as a function of the limb's orientation in the gravitational field (Bernstein, 1967).

\section{Task Constraints}

There have been many attempts to classify motor tasks on the basis of either fundamental principles or task similarities, such as Poulton's (1957) open skills and closed skills classification that is based on a continuum of environmental change. Consistent with our theme of adaptation to task constraints, it is possible to classify motor tasks in relation to three types of task constraint (Newell, 1986). The first is rule-constrained tasks, in which the performer is constrained in achieving a task goal by rules that constrain the nature of the movement output. Successful performance in such tasks requires attainment of a defined goal in a manner that is consistent with observing the rules. In such tasks, the biomechanical characteristics of the movement that is necessary to attain the goal are relatively unimportant, and, therefore, constraints on the coordination and control of limb movements are relatively few. Most ball games fall into this classification in that, although there are elaborate rules governing the overall conduct of the game, participants are relatively unconstrained in the manner in which they move their limbs to achieve the task goal. In Nelson's (1983) analysis, introduced above, performance objectives were defined in a way that was analogous to rules. For example, with distance fixed, the objective (or rule) might be to minimize movement time, as in a footrace.

A second classification is machine- and implementconstrained tasks, in which implements or machines are used, such as tools and vehicles. Such devices constrain the response very directly by imposing biomechanical restrictions on the operator. A bicycle, for example, constrains the legs to circular motion; the upper body is also, to a lesser extent, constrained by the design of the vehicle.

Finally, we can define biomechanically constrained tasks. When the motor response does not involve an implement, such as a tool or a machine, we consider the biomechanical constraints that define an activity as the task. In locomotor activities, such as running, walking, and swimming, the constraints on limb movements that define these activities are the task. For example, walking can be distinguished from running by the constraint that, in walking, one foot must be in contact with the surface at all times. In some sporting activities, stringent rules are applied to define biomechanically constrained tasks; in breaking those rules, the performer gains an advantage by being released from constraint. For some motor activities, the constraints are absolute; in gymnastics, for example, vaults and other accomplishments are defined solely by the ability to constrain limb movements to a specific movement pattern.

\section{A CONSTRAINTS-BASED FRAMEWORK FOR MOVEMENT ECONOMY}

In Figure 1, a conceptual framework is presented in order to show the interaction of the various constraints 


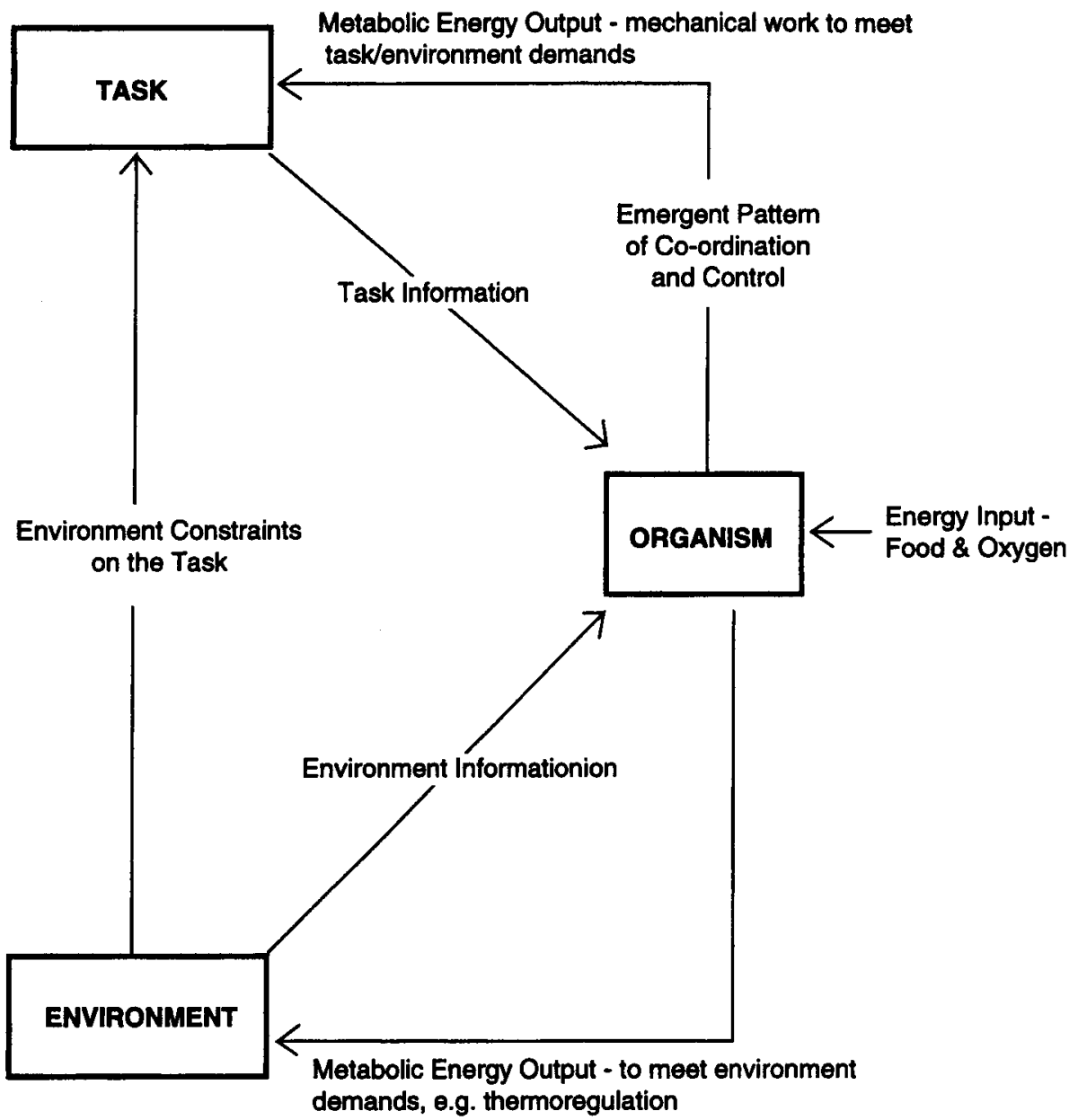

Figure 1. A constraints-based framework of metabolic energy expenditure and motor coordination and control.

that we described above and to show how they influence movement economy. An organism's movements are characterized as emerging from the interaction of environment and task constraints with constraints of the organism. It is proposed, furthermore, that the process of adaptation is guided by minimum metabolic energy criteria, so that task and environment constraints are accommodated with minimum metabolic cost. In addition to intrinsic sensory information about the state of the body, informational support for metabolic energy regulation is provided by the task and the environment. The components of the scheme presented in Figure 1 are described in detail below, whereas further discussion of the informational support for metabolic energy expenditure is presented in a later section.

\section{Metabolic Energy Input-Food}

Metabolic energy is input from food and oxygen, and this energy is converted to mechanical work and heat. Two main sources of metabolic energy output, or expenditure, can be considered: metabolic energy output to meet task demands and metabolic energy expenditure to meet environment demands.

\section{Environment Constraints on the Task}

In addition to being constrained directly by the task and the environment, the organism is also constrained indirectly by environmental influences on the task. When we refer to task constraints from now on, we will, therefore, also be including constraints imposed on the task by the environment. As an example of these interactions, consider the task of riding a bicycle, given the environment constraint associated with changes in terrain. In this case, the performer is required to adapt to environmental change, such as cycling uphill or downhill or cycling on rough or smooth roads. In such circumstances, environment constraints are experienced only indirectly and are accommodated through the task. When performing tasks defined by rules governing biomechanical constraints, interactions with the environment, such as running over undulating terrain, present the same type of environmental constraint as those encountered in implement- 
constrained tasks. We now proceed by reviewing evidence to show how movement patterns are adapted to task and environment constraints, consistent with the principles of economy of metabolic energy expenditure. Although in Figure 1 task and environment constraints are identified as separate components, we will consider how they influence movement coordination and control collectively as task/environment constraints. In practice, task and environment constraints interact, and there have been few attempts to examine their separate effects.

\section{MOTOR ADAPTATION TO TASK/ENVIRONMENT CONSTRAINTS}

In this section, evidence is presented to show how movements emerge as economical adaptations to task/ environment constraints. The literature concerning such adaptations is organized into four major subdomains: self-optimization, entrainment, movement economy and animal learning, and movement economy and human learning.

\section{Studies of Self-Optimization}

It has been shown that, in the short term, organisms naturally adopt a movement pattern that minimizes metabolic energy expenditure. This process has been referred to as self-optimization, which suggests that economical movements can be established without augmented information about the performer's cardiorespiratory response to exercise. In this section, we review studies that have demonstrated the self-optimization phenomenon across a range of motor tasks, but we have also provided an extended discussion of both human and nonhuman gait as a model case of how movements are coordinated and controlled in such a way that metabolic energy costs are minimized.

Sparrow (1983) reviewed several self-optimization studies of metabolic energy expenditure in arm-cranking, pumping, and other simulated work-type activities. When the subjects were asked to work at a freely chosen rate and, subsequently, at slower or faster tempos, it was observed that they were able to achieve the most economical tempo without augmented economy information from the experimenter. The data in Figure 2 from Salvendy (1972) demonstrate that, at freely chosen rates, subjects maximized their efficiency when riding a bicycle ergometer or operating a pump ergometer and maximized economy in bench stepping. In each case, the freely chosen rate falls between the two most extreme data points, so the conclusion does not depend on the accuracy of the theoretical curves in the figure. Performance was optimized at freely chosen work rates because, at movement cycle frequencies either faster or slower than the preferred rate, metabolic cost relative to work output was increased. It is important to note, however, that self-optimization occurs with respect to parameters other than work rate. For example, Nordeen-Snyder (1977) manipulated bicycle seat height, with time to complete a predetermined amount of work and oxygen consumption as the dependent variables. As in Salvendy's experiments, the subjects were able to control movements to accommodate task constraints, so that economy is maximized by selecting the seat height that corresponds to the most economical expenditure of metabolic energy.

Figure 3 presents data from Cavanagh and Williams (1982) that are characteristic of studies of self-optimization in the control of gait. Freely chosen stride length was first established, after which the subjects were constrained to run with stride lengths longer and shorter than the freely chosen lengths, with stride rate fixed. Figure 3 shows that stride length was self-optimized at a given running speed, in that it closely approximated the optimal stride length corresponding to the lowest and, therefore, most economical point on the oxygen consumption curve. Holt, Jeng, Ratcliffe, and Hamill (1995) were also interested in the relationship between metabolic energy expenditure and preferred stride characteristics. They adopted a $d y$ namical approach in which preferred coordination and control in human gait is associated with stability of body segments' trajectories over a stride cycle, and it was hypothesized that at preferred stride rate stability would be maximized and metabolic energy expenditure minimized. When subjects were asked to walk at preferred stride rates and at rates faster and slower than preferred, stability of the head trajectory, as measured by the standard deviation of the head's vertical displacement, was high and metabolic energy expenditure was minimized, a result that is consistent with Cavanagh and Williams's (1982) observations on the economy of preferred gait parameters. Holt et al. concluded by suggesting that the two most likely explanations for their findings were either that humans are sensitive to metabolic cost and adopt a preferred gait on the basis of such sensory information or that, consistent with dynamical systems theory, preferred behaviors emerge from stability considerations and an economical movement pattern is the consequence of stable dynamics. As Holt et al. made clear, the results of their study did not provide a critical test of the stability hypothesis, and they concluded by suggesting a combined self-optimization/dynamical systems approach to understanding the constraints on movement. In summary, although it is reasonable to hypothesize that preferred modes are dynamically stable, the question remains as to the physiological processes that allow us to select preferred modes. As the research findings stand, however, there are good grounds for suggesting that it would be fruitful to test further the hypothesis that gait parameters such as stride length and frequency are optimized on the basis of metabolic energy expenditure.

A further interesting manifestation of self-optimization is the abrupt transitions in coordination with short-term changes, or scaling, of the task constraints. In this case, our focus is not the refinement of an existing coordination pattern, as described above, but a fundamental change in the manner in which either individual limbs or limb segments within a limb are coordinated. When task metabolic demands reach a critical point, the existing coordi- 

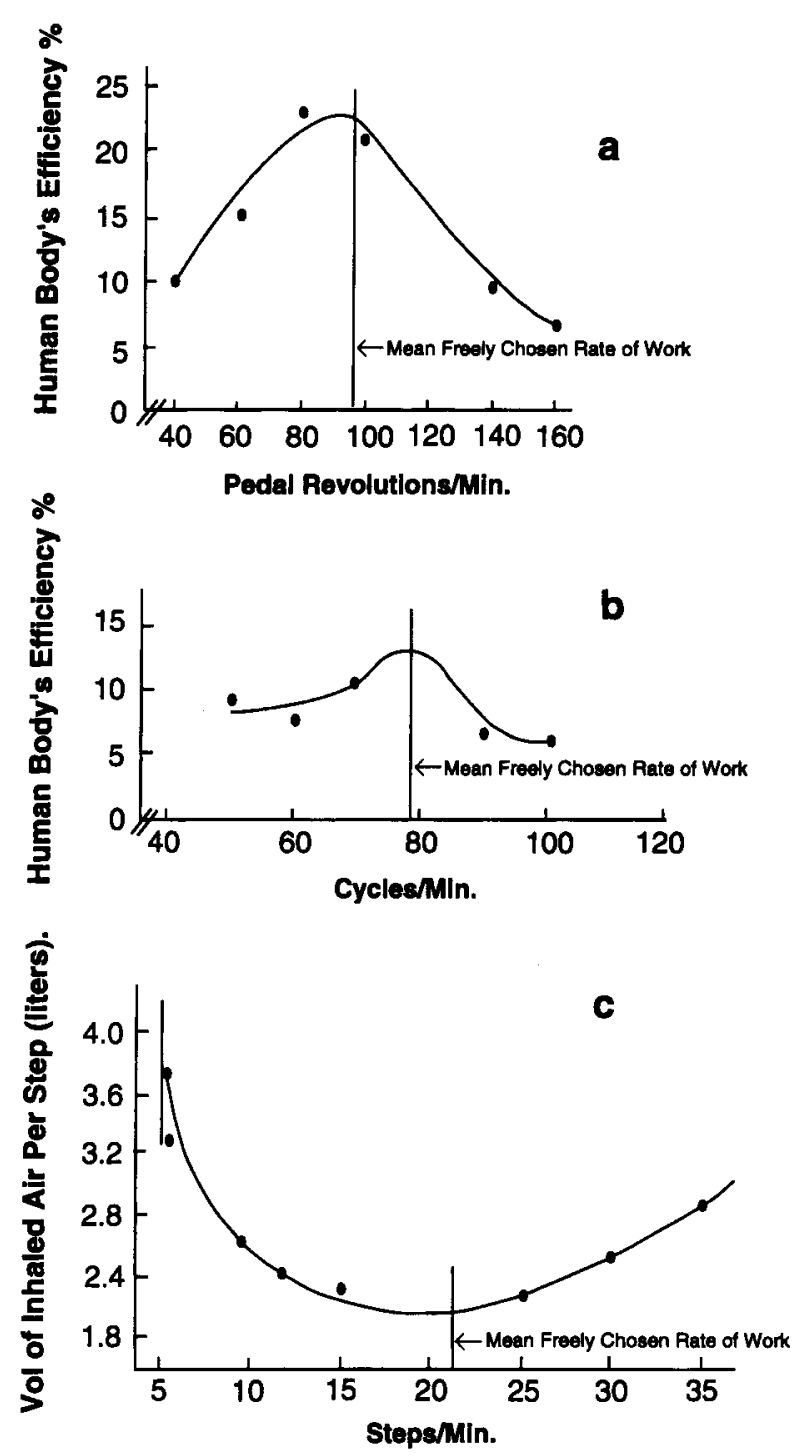

Figure 2. (a) Effect of paced and unpaced work on efficiency of the human body. Bicycle ergometer $(x: n=3)$. (b) Effect of paced and unpaced work on efficiency of the human body. Pump ergometer $(x: n=5)$. (c) Changes in the volume of inhaled air per step on the Harvard Step Test at paced and unpaced rate of work $(n=14)$. (From "Physiological and Psychological Aspects of Paced Performance," by G. Salvendy, 1972, Acta Physiologica Academiae Scientiarum Hungaricae, 42, p. 259. Reprinted with permission.)

nation pattern can, in some cases, become too costly, and the organism switches to a more economical coordination mode. Such abrupt spontaneous transitions in limb coordination have been shown in gait patterns by Hoyt and Taylor (1981), who trained horses to run on a treadmill and to change gait on command. The curves at the top of Figure 4 show the effect of an increase in speed on the metabolic energy expenditure of horses running on a treadmill. For each gait (walk, trot, or gallop), there was a speed at which metabolic energy expenditure was mini-

mized. The histograms at the bottom of Figure 4 show the speeds naturally selected by one horse while observed running on the ground. These observations highlight the self-optimizing phenomenon described above, because, in natural conditions, the horse chose the three speeds that corresponded to low oxygen consumption. The transition between gaits in the natural environment also occurred close to where the oxygen consumption curves at the top of the figure intersect. In addition, as Hoyt and Taylor pointed out, whereas on a motorized treadmill the animal must move at the speed of the tread, when running on the ground, there were ranges of speed that the animal never used for any sustained period, as the figure shows. The authors concluded that these data support the hypothesis that the interlimb coordination pattern, characteristic of each gait, is changed in order to minimize metabolic cost.

It can also be observed that the oxygen consumption curves in Figure 4 have approximately the same minimum, about $15 \mathrm{ml}$. This means that the minimum energy expended to move $1 \mathrm{~m}$ is independent of the gait mode. Although Hoyt and Taylor (1981) did not comment on this observation, it is possible that change in gait not only minimizes oxygen consumption but also maintains the oxygen cost of coordinating the limbs constant per unit distance traveled. Thus, oxygen cost could be hypothesized to be an invariant that constrains an organism's limb movements to a movement pattern that does not exceed a metabolic threshold. In human runners, the metabolic cost per unit distance traveled also remains constant with an increase in speed (Margaria, Cerretelli, Aghemo, \& Sassi, 1963).

Further parallels in the findings from the research on human and nonhuman subjects can be found in a paper on stair climbing by Warren (1984) and, more recently, Diedrich and Warren's (1995) study of the walk-run transition in human gait. In his stair-climbing paper, Warren proposed that transitions in movement coordination patterns take place at critical points and suggested that, if stair-riser height was, hypothetically, increased too far to afford bipedal ascent, at the critical point a four-limbed creeping, or climbing, gait would be adopted. Such a critical point for riser height in stair ascent is essentially the same, in principle, as the critical locomotor speed for animal gait transitions described above. Diedrich and Warren suggested, however, that the energy minimization explanation for gait transitions is not unequivocally supported; nevertheless, they cited Falls and Humphrey (1976) and Margaria (1976), who did show a close correspondence between the walk-run transition speed and the metabolic energy demanding optimal speed. In contrast, Hreljac (1993) indicated that, at the transition speed, the energetic cost of running was $16 \%$ higher than that for walking, but Diedrich and Warren also added that the runner's acceleration would "soon act to reduce energetic costs" (p. 184). Diedrich and Warren's experiments were predicated on a dynamic theory of the walk-run transition, with qualitative change in the movement dy- 


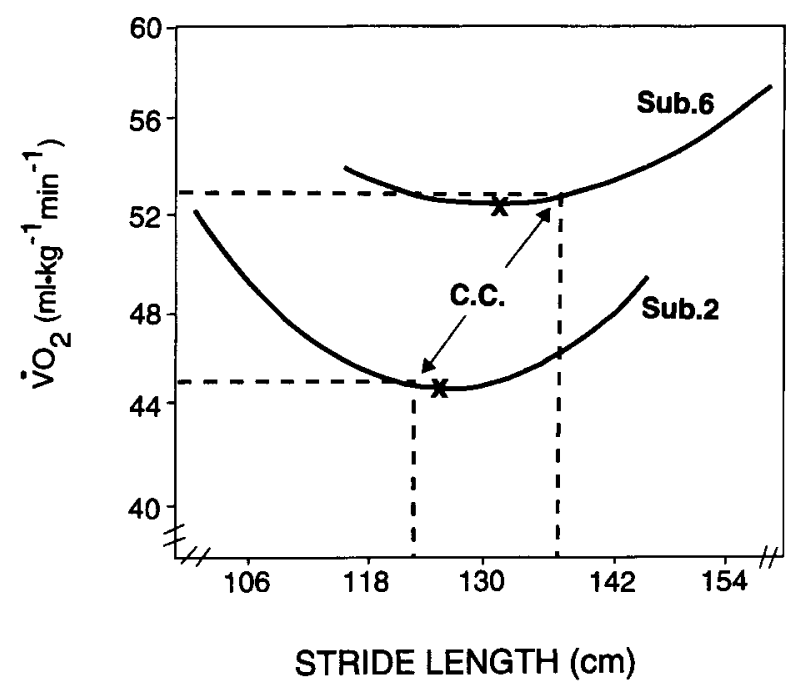

Figure 3. Best-fit curves for 2 subjects showing the relationship between oxygen consumption and stride length. The crosses on each curve represent the predicted stride length at which economy would be maximized, and the dashed lines show the subjectchosen optimum conditions (C.C.) Subject 2 shows a smaller $\mathrm{O}_{2}$ uptake and shorter stride lengths than does Subject 6. (From "The Effect of Stride Length Variation on Oxygen Uptake During Distance Running," by P. R. Cavanagh and K. R. Williams, 1982, Medicine and Science in Sports and Exercise, 14, p. 32. Copyright 1984 by Williams \& Wilkins. Reprinted with permission.)

namics, which is characteristic of phase transitions in self-organizing systems in general, occurring when the system becomes unstable. Lack of stability and the associated abrupt transition to a new dynamically stable state, such as a running gait, were hypothesized as the underlying cause of the shift, with energetic considerations "not assumed to be the proximal cause of a transition" (p. 184). There are, however, three important considerations in evaluating the dynamic theory as a rival hypothesis. First, Diedrich and Warren's experiments did not measure metabolic energy expenditure to confirm, or otherwise, the findings cited above concerning the relative metabolic cost of walking and running at the transition. Second, unlike the gait transitions observed in quadrupeds, the walk-run transition in human gait is not associated with a change in interlimb timing relationships, in that the feet continue to maintain a half-cycle phase difference. Third, in evaluating their proposal that "perceived exertion could be more closely related to the dynamics of the task than to the overall energetics" (p. 198), it should be noted that, in tasks such as running and cycling, there are strong positive correlations between ratings of perceived exertion, heart rate, and oxygen consumption (Borg, 1973). Thus, as with the selection of preferred gait parameters discussed above, our discussion highlights an interesting and important question - that is, the nature of the trigger for gait transitions.

In a study in which human subjects adopted a typically nonhuman four-limbed gait, Sparrow and Newell (1994) measured oxygen consumption in treadmill walking and creeping (walking on hands and feet) at progressively greater positive grades. The oxygen consumption curves for the two activities converged as grade increased, as is indicated in Figure 5. We hypothesized that, if treadmill grade could have been increased further, the point at which they crossed would be a critical point at which the subject would have switched from upright walking to a fourlimbed creeping gait. These data, therefore, further support the view that abrupt transitions in movement patterns at critical points are due to organizational changes that confer greater economy of metabolic energy expenditure.

As a footnote to this section, it is important to point out that recent theorizing about the energetics of transitions in coordination has emphasized the self-organizing properties of physical systems in general, with gait transitions considered to be just one type of spontaneous shift in movement organization. In such theoretical work, the phase relation between the limbs is referred to as the order parameter, which changes abruptly because of the lack of stability engendered by an increase in speed (Haken, Kelso, \& Bunz, 1985). It is, however, interesting to speculate further as to the nature of the variable underlying the transition, because changes in locomotor speed are also associated with variations in metabolic parameters, and, as highlighted in our discussion of Diedrich and Warren's (1995) paper, the nature of the key quantity that precipitates transitions has not been conclusively demonstrated. The major difficulty in resolving this issue will be providing a critical test of the causal relationship between either metabolic variables or biomechanical measures of movement pattern stability and the timing of gait transitions.

\section{Entrainment}

The term entrainment refers to the degree of consistency in temporal phasing between physiological variables and specified events of a movement cycle. Since the first quarter of the century, links between internal metabolic processes and rhythmic or cyclical movements have been observed. Coleman (1921), for example, noted relationships between pulse rate, rate of stepping, and breathing rate in captive animals in zoological gardens. He also showed a relationship between the animals' respiration and walking, so that there was a fixed ratio between the number of steps and the number of breaths. Patterson (1916) and Seashore (1926) also observed correspondence between motor responses and physiological adaptations such as changes in heart rate and respiratory rate.

Recent investigations have revealed the same phenomenon with more rigorous contemporary methodology. Some studies have shown entrainment (or coupling) of rowing, pedaling, or gait frequency with breathing (Bannister, Cunningham, \& Douglas, 1954; Bechbache, Chow, Duffin, \& Orsini, 1979; Bechbache \& Duffin, 1977; Mahler, Shuhart, Brew, \& Stukel, 1991; Steinacker, Both, \& Whipp, 1993). There have, however, been disagreements as to the cause and generality of these findings. Kay, Petersen, and Vejby-Christensen (1975), for exam- 


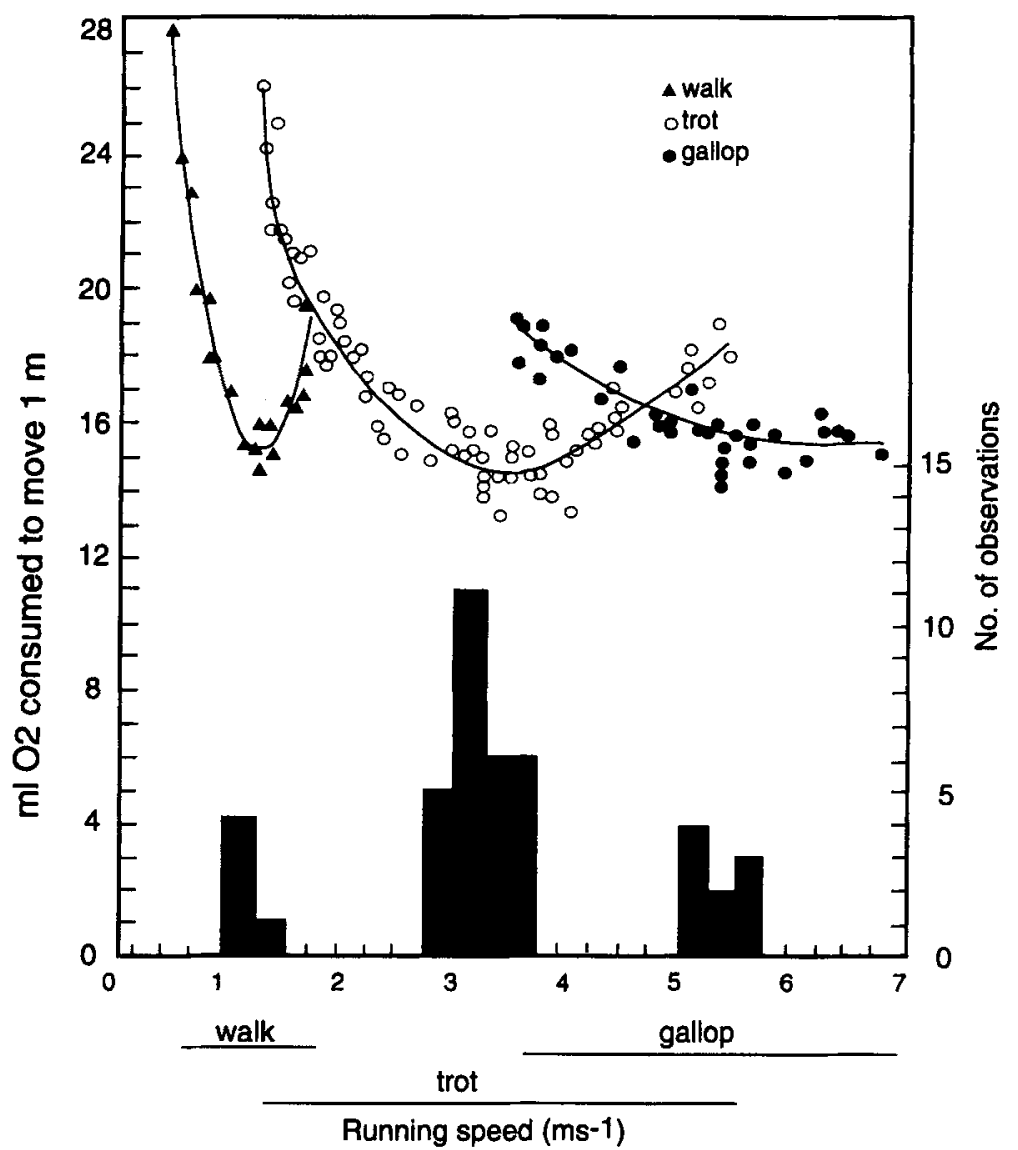

\begin{abstract}
Figure 4. The oxygen cost to move a unit distance (rate of oxygen consumption divided by speed) for horses walking, trotting, and galloping on a motorized treadmill. The histogram shows gaits when one horse was allowed to select her own speed while running on the ground. She chose three speeds that coincided with the energetically optimal speed for each gait. (From "Gait and the Energetics of Locomotion in Horses," by D. F. Hoyt and C. R. Taylor, 1981, Nature, 292, p. 240. Copyright 1981 by Macmillan Magazines Ltd. Reprinted with permission.)
\end{abstract}

ple, did not find evidence to support the hypothesis that breath duration is a submultiple of either stride frequency or pedaling frequency. They suggested, furthermore, that the earlier Bannister et al. findings might have been influenced by the procedure of asking the subjects to stride in time with a metronome.

Later, however, Bramble and Carrier (1983) did reveal reliable phase relationships between breathing rate and frequency of limb movements. They showed that some quadrupeds establish less variable stride-to-breath ratios than do human runners, usually one stride per breath (1:1), whereas human subjects adopt ratios ranging from $1: 1$ to $4: 1$. Bramble and Carrier summarized the relationship between gait, breathing, and energy regulation by saying that, "The inability of humans to change gait while running implies that their exceptional capacity to alter breathing pattern could to some extent represent an alternative strategy for regulating energetic cost" (p. 255). Thus, in contrast to quadrupedal animals, there is no fundamental change in the interlimb coordination pattern of human gait when the mechanical power output rises as a consequence of increased speed.

Kirby, Nugent, Marlow, MacLeod, and Marble (1989) also found, in a study of both cycling and walking, that limb movements and heart rate were coupled, or entrained, in 18 of 25 subjects at a range of gait and pedaling speeds. The cadence and heart rate data for 1 subject are shown in Figure 6. Some subjects also had the capacity to reestablish coupling when it was disrupted by slowing the treadmill. With the caveat that some subjects did not entrain and that, for others, entrainment was speed and task dependent, the study provides further evidence to support the hypothesis of a relationship between motor output and the timing of cardiovascular responses to exercise. Kirby et al. also raised the question, without providing an answer, as to the relationship between cadence and heart rate coupling, mechanical work, and metabolic cost. Our interpretation, which is consistent with Bramble and Carrier (1983), is that entrainment is related to the organism's propensity to reduce metabolic energy ex- 

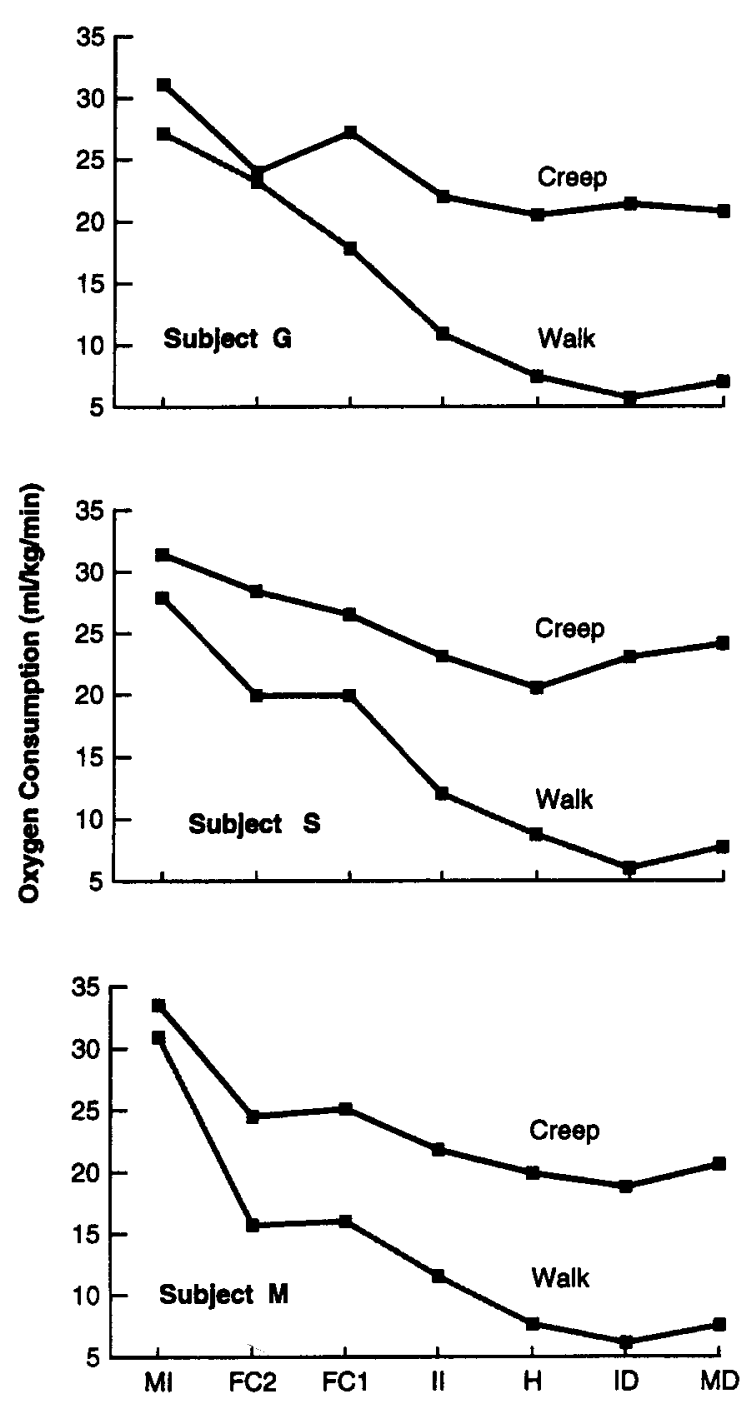

TREADMILL GRADE

Figure 5. Oxygen consumption for 3 adult males when either walking or creeping at an average speed of $2.4 \mathrm{kph}(1.5 \mathrm{mph})$ on a motor-driven treadmill at positive and negative grades. MI, maximum incline $(+29 \%)$; FC, freely chosen grade; II, intermediate incline $(+8 \%) ; \mathrm{H}$, horizontal $(0 \%)$; ID, intermediate decline $(-8 \%)$; MD, maximum decline $(-18 \%)$. (From "Energy Expenditure and Motor Performance Relationships in Humans Learning a Motor Task," by W. A. Sparrow and K. M. Newell, 1994, Psychophysiology, 31, p. 344. Copyright 1994 by Cambridge University Press. Reprinted with permission.)

penditure in response to internal feedback mediating cardiovascular responses.

More recently, the above findings have received attention from those interested in the physics of self-organizing systems, with the frequency locking that Bramble and Carrier (1983) observed shown to be predictable from mathematical models (Beek, Peper, \& van Wieringen, 1992). Specifically, contemporary movement science theorists have been interested in the association between frequency locking, or synchronization, and dynamic stability, so that losses of stability are associated with abrupt and discrete shifts or bifurcations in coordination, as in shifting from one breathing-to-stride-frequency ratio to another. Of particular importance for our metabolic energy minimization hypothesis is the finding that the movement stability associated with entrainment is, as Beek et al. phrased it, "often associated with the minimization of some energy cost function" (p. 603), an observation that is consistent with Bramble and Carrier's conclusion concerning the energy cost strategy, cited above. Most contemporary work on entrainment, energy cost, and transitions to new stable states has not measured metabolic energy cost directly; rather, it has been inferred that metabolic energy expenditure is minimized when frequency locking is stable. As noted earlier, however, the emerging literature on dynamic stability and transitions in human gait reveals increasing interest in the association between stability in movement mechanics and metabolic energy cost.

Although the focus of this section is entrainment, with practice issues to be considered later, it is convenient to note here that Bramble and Carrier (1983) also showed that, with experienced human runners, the coupling of breath to stride was more precise than with inexperienced runners. Individuals who trained seriously for swimming were also less able to maintain the tight coupling of respiration to stride pattern, even though their gait showed no kinematic irregularities. This suggests that, in some activities, the capacity to synchronize the movement pattern and physiological responses to exercise might require extensive practice. The issue of practice effects on motor economy is considered in the following section.

\section{Movement Economy and Animal Learning}

Learning experiments with rats early in the history of experimental psychology laid the foundations for later theorizing about the learning and control of motor responses in humans (Adams, 1968; Keele, 1968). There is, however, a literature on economy and learning that has been largely overlooked by motor-learning theorists. This work has its origins in experiments on minimum principles in animal learning that were undertaken by De Camp (1920), Kuo (1922), Sams and Tolman (1929), and others in the 1920s and later by investigators such as Tsai (1932), Waters (1937), and McCulloch (1934). The major principle that emerges from this literature is that, with practice, organisms learn to adapt movements in order to achieve the task goal with the least metabolic energy expenditure and, therefore, greater economy. Tolman (1932) distilled these findings in his book Purposive Behaviour in Animals and Men and generated the principle of least effort to account for the experimental animal's propensity to seek reward on the basis of the distance, time, or force involved. Further support for the least effort hypothesis was provided by Solomon (1948), who reviewed a number of principles related to work and behavior, empha- 


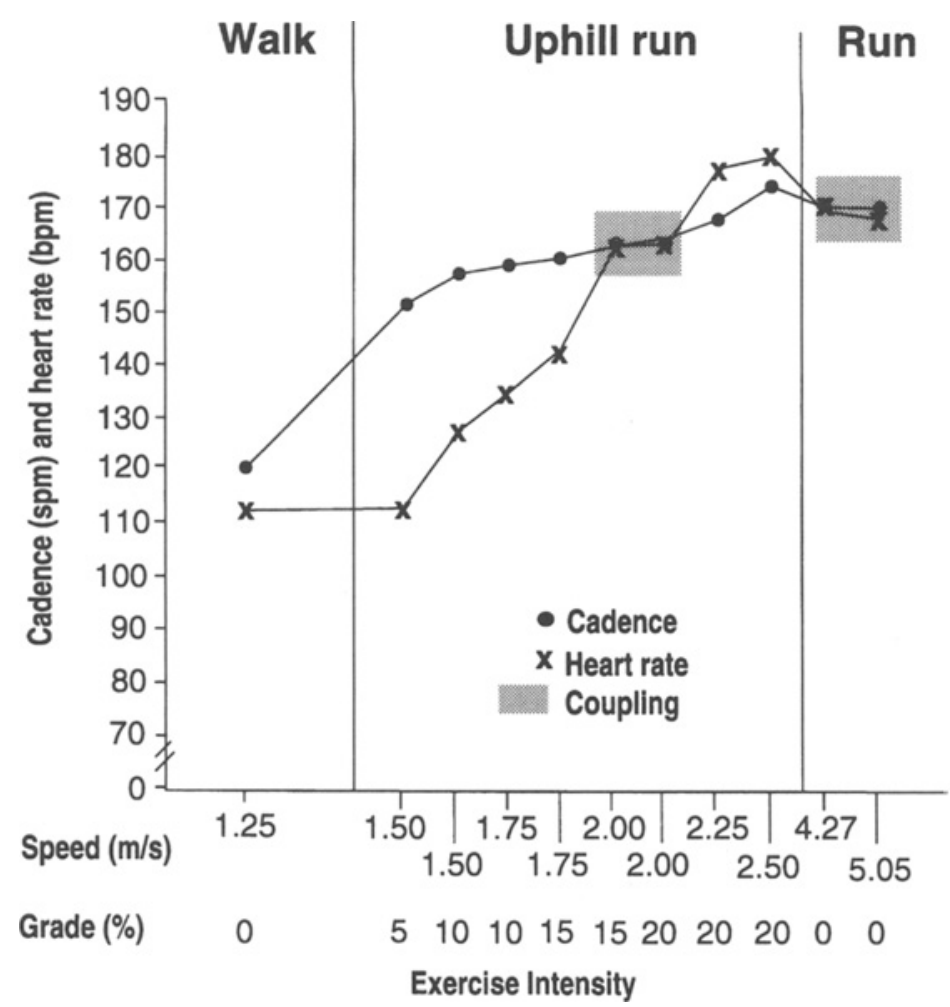

Figure 6. Cadence in steps per minute (spm) and heart rate in beats per minute (bpm) plotted against exercise intensity for 1 subject walking, running uphill, and running on the level. Coupling, indicated by stippled rectangles, was found at two uphill-running and two level-running intensities. (From "Coupling of Cardiac and Locomotor Rhythms," by R. L. Kirby, S. T. Nugent, R. W. Marlow, D. A. MacLeod, \& A. E. Marble, 1989, Journal of Applied Physiology, 66, p. 326. Copyright 1989 by The American Physiological Society. Reprinted with permission.)

sizing Hull's interest in earlier investigations of minimum effort in maze learning by rats. The amount of effort or work involved in responding was given a central role in Hull's (1943) Principles of Behavior.

Hull (1943) proposed that, with repeated responding, motivation to cease the activity develops, akin to fatigue. He called this reactive inhibition, abbreviated IR. The amount of IR produced was said to increase as a function of the amount of work and to dissipate on cessation of the activity. Closely associated with IR is the learned response of not responding, called conditioned inhibition (SIR). Failure to respond takes on rewarding properties by virtue of the relief from the distress associated with responding repeatedly. Thus, a response requiring little effort will lead to less response-inhibiting stimulation and gradually dominate more effortful responses. Hull stated in his law of less work:

If two or more behavior sequences, each involving a different amount of energy consumption or work (W), have been equally well reinforced an equal number of times, the organism will gradually learn to choose the less laborious behavior sequence leading to the attainment of the reinforcing state of affairs. (p. 294)
Hull (1943) made explicit the proposition that mechanical work (the product of force and distance) is the variable causing the (unobservable) inhibiting potential to produce a cessation of activity. In his statement of the law of less work, quoted earlier, Hull also referred to energy consumption, but, unlike work, this variable was neither defined operationally nor included in the systematic development of his corollaries. The lack of operational definition occurred, perhaps, because there were no practical procedures for measuring an animal's metabolic energy consumption in the tasks that he studied. The reference to energy consumption probably was made because experiments using force-resisting devices measured directly the mechanical work output of the response, whereas experiments involving ambulatory responses could not provide a direct measure of mechanical work; therefore, energy consumption was proposed as the less work variable.

After the 1940s, there appears to have been little further exploration of economy and motor learning. Certainly no attempts were made to develop a principle of least effort along the lines suggested by Tolman (1932). Perhaps it was the decline of interest in maze learning that shifted 


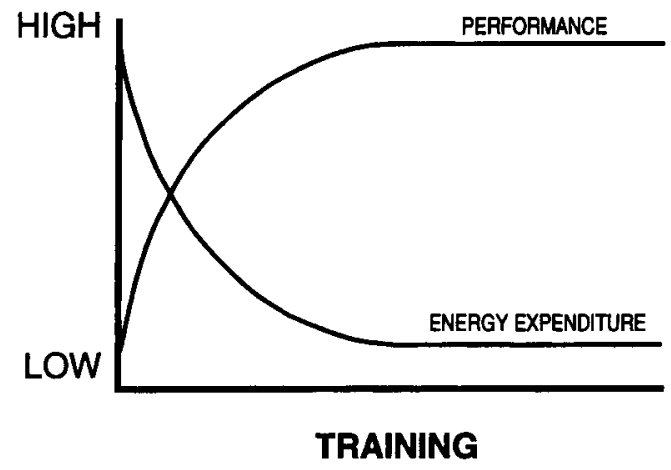

Figure 7. Hypothesized alterations in energy expenditure during skill learning (after Freeman, 1948, and Sparrow, 1983). (From "Behavioural Energetics: Some Effects of Uncertainty on the Mobilization and Distribution of Energy," by J. Brener, 1987, Psychophysiology, 24, p. 503. Copyright 1987 by Cambridge University Press. Reprinted with permission.)

the focus of comparative psychologists away from such high-energy-demanding activities. Another possibility is that sufficient empirical work had been carried out to bring the issue to a close within the bounds of the extant theory. Time, distance, and force, had been examined, possible confounding variables had been considered - such as the attendant delay of reinforcement in longer paths (Sams \& Tolman, 1929) - and the threshold of discriminability of routes of varying length had been established by Yoshioka (1929) and Thompson (1944). Whatever the reasons, later animal-learning experimentation was dominated by the Skinnerian tradition of response rate as the dependent variable, and issues related to movement economy disappeared from the literature.

Tolman (1932) concluded his discussion of meansend-distances in maze learning by saying that the animal's preference for the shorter, temporally shorter, or least force-resisting route may be "reducible to a single, more fundamental, one - to be called the preference for "Least Effort"' (p. 110). He also raised the possibility of a single physiological principle subserving such least effort responses. Tolman was not explicit about the nature of such a physiological principle but probably would not have been antipathetic to the proposal that metabolic energy expenditure could form the basis of a principle of least effort. Our conclusion from Tolman's work and from the other studies reviewed above is that metabolic energy expenditure rather than mechanical work is the fundamental variable underlying all of the factors that were manipulated.

Learning theorists using nonhuman subjects showed little interest in principles related to movement economy during the 1950s. In the 1960s, however, issues related to learning and the energetics of response reappeared in the work of Notterman and Mintz (1965). In these experiments, a strain gauge was attached to a manipulandum so that the force/time characteristics of the rats' responses could be measured. With this equipment, various rein- forcement criteria could be specified, such as peak force, response duration, or the time integral of force. In addition, unlike the traditional operant learning equipment, this device permitted the measurement of responses that did not reach the reinforcement criterion. The data showed that rats could learn to discriminate the response cues necessary to achieve the criterion force, time, or forcetime. Although Notterman and Mintz introduced their series of experiments with a discussion of the relationship between mechanical work and metabolic energy, they did not measure metabolic energy expenditure directly. In contemporary operant-learning work by Brener (1986), however, direct measures of metabolic energy expenditure have been used in theorizing about the relationship between metabolic energy expenditure and learning.

Brener's (1986) position is that behavioral economy is a consequence of eliminating components of the response that are not reinforced, while strengthening those that are. Brener also shows some sympathy for the ecologists' position in discussing the evolutionary rationale for learning that is guided by considerations of economy. For example, Krebs (1978) reviewed findings on optimal foraging, in which the net energy gain from prey of a certain type and size is, in part, dependent on the distance traveled associated with the animal's search strategy. Krebs' discussion of the modification of search paths with experience reinforces the findings from the least effort experiments, discussed earlier. Thus, the findings from the early learning experiments and the search-economy predictions of optimal foraging theory support Brener's proposition that behavioral efficiency is reinforced by the biological utility of its consequences. In an experimental investigation of this proposition, Brener and his colleagues (Brener, Phillips, \& Sherwood, 1983) tested the hypothesis that animals will maximize the net energy gains from food by reducing the metabolic energy expended in its attainment. An experimental group of rats ran for a food reward, whereas a second group was a yolked control fed at the same frequency as the experimental group, independent of their ambulatory behavior. Contrary to the energy maximization principle, the control group did not differ from the experimental group in running rate, oxygen consumption, or heart rate. The proposed explanation for this was similar to Notterman and Mintz's (1965) biological explanation, in that the natural tendency of rats to run to attain food when hungry could account for the control group's unexpectedly high ambulation rate. Brener et al. also expressed doubt about the assumption that running "would constitute a reliable and prominent source of energy expenditure in the experimental situation" (p. 390), because the energy cost of ambulation was fairly low, as compared with the calorific value of the food reward. This implies that the animals were insensitive to the relationship between the calorie value of the food and the amount of metabolic energy expended.

In later work, Brener and Mitchell (1989) adapted Notterman and Mintz's (1965) protocol, which revealed that, over days of training, rats learn to produce a peak 
force to gain food reinforcements. Brener and Mitchell's integrated force/time values decreased reliably over days, even though the peak forces were, overall, $73 \%$ greater than the reinforcement criterion. Brener and Mitchell also recorded the animal's rate of oxygen consumption inside the air-tight experimental box and found that oxygen consumption $(\mathrm{ml} / \mathrm{kg} / \mathrm{min})$ decreased reliably over days. Thus, Brener and Mitchell's findings support Sparrow (1983) and Freeman's (1948) hypothesized relationship between movement economy and performance as a function of practice, as is shown in Figure 7.

Further support for the hypothesized relationship observed in Figure 7 comes from training studies with human subjects. For this relationship to hold, task constraints would remain unchanged, and refinements to the movement pattern that are commensurate with reductions in metabolic energy expenditure would be anticipated. In the following section, we review the literature concerning reductions in metabolic energy expenditure in human learning with task constraints either fixed, revealing the function shown above, or systematically manipulated in order to show adaptations to new task constraints.

\section{Movement Economy and Human Learning}

In studies of human motor learning, little attention has been paid to the early minimization principles described above. Motor-learning research with humans through the 1940s and 1950s progressed on the basis of Hull's (1943) theorizing, but, for the most part, it was issues related to reactive inhibition and distribution of practice that drove the research enterprise. At about the same time, however, George Kingsley Zipf (1949), an ecologist, published a book titled Human Behavior and the Principle of Least Effort. A brief discussion of Zipf's work provides the proper starting point for this section on human learning, because of its historical continuity with the animallearning work and because of its importance in proposing that the least work principle underlies a broad range of human behavior. Although Zipf's analysis cannot be overlooked, it, like Freeman's (1948) work, lies outside the continuity of ideas that we have developed within the mainstream of experimental psychology and related disciplines. Zipf was concerned with the behavior of both individuals (individual economy) and societies (collective human economy). His fundamental thesis, which consistent with that of the animal-learning theorists, was that "every individual's entire behavior is governed by the Principle of Least Effort" (p. 6). Least effort was equated with work; he was, therefore, sympathetic to Hull's (1943) theory and made similar observations on the choice of least-work-demanding alternatives in everyday human behavior. He also extended the least effort principle to the question of how such considerations might influence a society's decisions on building a bridge and other such long-term work-saving decisions. The subtitle to Zipf's book was An Introduction to Human Ecology, and, in terms of contemporary disciplines, it could be described as having a human geography or macroeconomic flavor.
To support his theory, Zipf used such data as word frequency usage and frequency of telephone messages between cities. Although such data can reasonably be viewed as an indirect measure of effort or mechanical work, their interpretation is, philosophically, somewhat removed from our central theme of metabolic energy expenditure and attendant changes in limb movements. Although Zipf's book can be considered an important landmark in the development of ideas about minimum principles in human behavior, only in recent years has economy in motor learning by human subjects been investigated experimentally.

The first contemporary area of experimental work to be considered in this section is that concerning quantitative theories of skilled movements. This research departs somewhat from a primary focus on metabolic energy expenditure, because it emphasizes the economy of movement mechanics, independent of physiological measures. This literature is, nevertheless, important because, in common with the early learning theorists, the fundamentals of contemporary quantitative theories are that skilled movements not only achieve some behavioral objective but also have quantities that can be described in terms such as smooth, easy, or economical. These quantities have been operationalized in a number of analyses of simple movement trajectories, with minimum cost variables being defined in terms of time, force, impulse, energy, and jerk (the time derivative of acceleration; Nelson, 1983). Jerk in multijoint movements has been investigated by Hogan and Flash (1987); minimum torquechange has been another minimum principle studied (Uno, Kawato, \& Suzuki, 1989); least travel cost and least spatial error cost have been used to model the optimum kinematics of reaching movements (Rosenbaum, Loukopoulos, Meulenbroek, Vaughan, \& Engelbrecht, 1995). Rosenbaum and his colleagues have also developed the notion of postural comfort from experiments in which subjects were requested to provide psychophysical ratings of the effortfulness or comfort associated with various postures. Given the close association described elsewhere between measures of perceived exertion (Borg, 1973) and metabolic variables, it is possible that end comfort may also be associated with minimum metabolic cost. In one of our recently completed experiments, an association has, in fact, been demonstrated between changes in metabolic variables with practice and perceived exertion, measured with Borg's scales. With the task demands fixed, perceived exertion was shown to decrease reliably and systematically over days of practice, consistent with improvements in movement economy and a significant reduction in heart rate (Sparrow \& Hughes, 1997). Thus, measures of comfort or perceived exertion do appear to be related to changes both in the mechanics of movement, as measured in the quantitative theories approach, and in metabolic energy expenditure.

Another good example of the quantitative theories approach is a study by Wann (1987), who calculated minimum cost variables for children assessed as either poor or 
good handwriters. Impulse, force, power, and jerk estimates from an analysis of the letters $U$ and $W$ were all reliably greater for poor writers. Thus, as Wann (1987) concluded, the good writers were more efficient, in the sense that the objective, writing a $U$ or a W, would have been met with less energy expenditure. Similarly, Flash (1990) has speculated as to the physiological interpretation for the success of minimum cost theories in predicting movement trajectories. She assumed that there is a more fundamental process underlying the observed movement pattern, in that the minimum cost variable is not sensed or computationally derived by the nervous system. Once more, this raises the possibility that the individual's propensity for metabolic energy conservation underlies the observed minimum cost kinematic and kinetic measures.

Nelson (1983) applied the predictions from his minimum cost calculations to an analysis of kinematic data from violin bowing. He compared the movement patterns that minimized minimum impulse and minimum mechanical energy with the obtained velocity patterns of skilled violinists. The minimum cost curves fitted all the experimental data well, although the minimum energy pattern better fitted the faster bowing strokes than it did the slower strokes. These observations illustrate the general principle that well-practiced movements not only satisfy a task objective but also embody a quality of ease or economy, as defined using minimum cost criteria. Similarly, in a study of running economy, Lin (1980) developed minimum energy criteria for subject-specific anthropometric data, such as limb masses and lengths. He found that seven highly skilled runners closely approximated the optimal stride length at which metabolic energy expenditure would be minimized. Other such optimal programming approaches to human gait analysis have been undertaken on the basis of a performance criterion that minimizes work done (see Chow \& Jacobson, 1971, for a review).

Some articles have described the physics of various sports activities, such as Brancazio's (1981) study of basketball shooting. Brancazio's calculations identified an optimum shooting angle that required the smallest force and ensured a large margin for error in the ball trajectory, relative to the position of the basket. This study did not analyze actual basketball shots for comparison with the results of the analytical calculations. The author, nevertheless, concluded that skilled players appear frequently to shoot with trajectories that require minimum force and have a high probability of success, on the basis of his margin for error principles.

In addition to studies of skill and movement mechanics, there are also data to show that, with practice, individuals perform the same task with lower metabolic energy expenditure. Indeed, the phenomenon of motor efficiency (Whipp \& Wasserman, 1969) that is due to the effect of practice on metabolic energy expenditure under standard testing procedures has long been recognized by respiratory physiologists. It is interesting to note, however, that the processes of motor coordination that underlie such changes have never attracted attention within this domain. Even in an activity as well practiced as walking, slight decreases in metabolic energy expenditure have been observed over trials on a motor-driven treadmill (see, e.g., Bobbert, 1960). With practice at novel tasks, however, changes in coordination led to marked reductions in metabolic cost. Poole and Ross (1983), for example, showed that the average oxygen consumption for experienced sheep shearers (an average of 91 months) was $2.95 \mathrm{ml}$ per sheep, whereas inexperienced workers (an average of 12.5 months) consumed significantly more oxygen per sheep ( $4.46 \mathrm{ml})$. Similarly, work by Wyndham et al. (1966) revealed interindividual differences in the economy of shoveling and other tasks associated with mining. Wyndham et al. described workers who performed a standardized task with lower oxygen consumption as more skilled, and, interestingly, they also found that skilled workers, by this criterion, had lower intraindividual variation in oxygen consumption, as measured by day-to-day variations in performing the same standardized task.

As with entrainment and self-optimization, studies of the economy of human gait - of running, in particularhave provided interesting interindividual comparisons of skilled and unskilled performers. DeVries and Housh (1994), for example, presented a figure showing the oxygen consumption of 17 male runners during treadmill running at constant speed. There were intraindividual variations of up to $54 \%$ in oxygen consumption, with a champion marathoner recording $26 \mathrm{ml} / \mathrm{kg} / \mathrm{min}$ for the run and the least skilled runner, as measured by oxygen consumption, requiring $40 \mathrm{ml} / \mathrm{kg} / \mathrm{min}$. One question arising from these and similar findings is why individuals show wide variations in economy. A recent review by Anderson (1996) provides a good coverage of potential sources of interindividual variations in running economy. Consistent with our earlier discussion, Anderson identified the effect of anthropometric dimensions (our organism constraints) on running economy, and he also summarized the kinetic, kinematic, and muscle contraction timing characteristics of running economy. One limitation to Anderson's review, however, was the failure to address the question as to how economical movement patterns emerge. Movement kinetics, kinematics, and the optimal timing of the muscle's stretch-shortening cycle may, in part, reflect individual differences in anthropometric and physiological variables, but they are also likely to be influenced by practice.

The first demonstration of improvements in economy with practice was made by Salvendy and Pilitsis (1974), motivated by considerations of energy expenditure in repetitive manual work, as with Poole and Ross (1983) and Wyndham et al. (1966), above. In the section on selfoptimization, Salvendy's interest in the physiological aspects of pacing in repetitive work situations was addressed (Salvendy, 1972). Salvendy and Pilitsis extended these interests to the question of physiological measures of work stress that are sensitive to the operator's adaptation to work with practice. The 5 subjects in their experiment repetitively moved and positioned a 3.5 -lb. weight either 


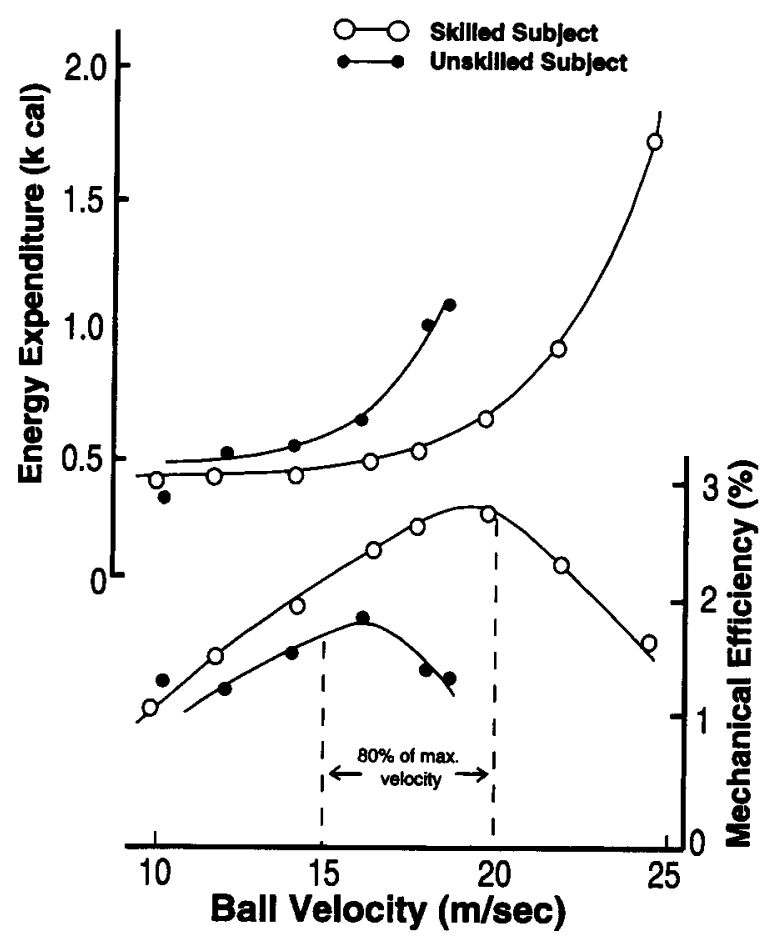

Figure 8. The relationship between ball velocity, energy expenditure, mechanical efficiency, and kicking accuracy for a skilled and an unskilled soccer player. (From " Energy Efficiency of Ball Kicking" by T. Asami, H. Togari, T. Kikuchi, N. Adachi, K. Yamamoto, K. Kitagawa, \& Y. Sano. In P. V. Komi (Ed.), Biomechanics (Vol. 13, p. 137). Baltimore, MD: University Park Press, 1976. Copyright 1976. Reprinted with permission.)

one-handed (left and right hand), or two-handed (i.e., both hands simultaneously). In the course of 1,000 trials (movement cycles), all of the subjects reduced the metabolic cost per cycle of transporting and positioning the weight. There were also systematic reductions in cycle time over trials, indicating that speed of performance improved concomitant with the improvements in economy. This investigation demonstrated, therefore, that, with expired air techniques, metabolic energy expenditure could be shown to decrease when the task constraints of moving an object of fixed mass a constant distance were unchanged. The reduction in cycle time also indicated that, when a performance criterion other than metabolic economy was employed, performance also improved, as measured by that (dependent) variable.

From a human motor-learning point of view, however, perhaps the most intriguing study of movement economy is that by Asami et al. (1976) that examined the relationship between skill level, metabolic energy expenditure, and accuracy of outcome on a ball-kicking task. The significance of this experiment lies in the fact that skill, economy, and performance measured with traditional speed/accuracy measures were shown to be interrelated. The curves in Figure 8 for a skilled subject and an unskilled subject show the relationship between metabolic energy expenditure, the velocity of a soccer ball kicked at a target, and mechanical efficiency, calculated by dividing kinetic energy imparted to the ball by net metabolic energy expenditure. From the top two curves in Figure 8, it is clear that the metabolic energy expenditure required to kick the ball was less for the skilled subject, and, as shown in the bottom graph, mechanical efficiency was greater for the skilled performer. When kicking accuracy was measured, it was found that maximum accuracy was achieved, for both samples combined, at $80 \%$ of maximum velocity. Curiously, this is the percentage of maximum velocity at which mechanical efficiency was maximized. The broken lines in Figure 8 (which we have added) have been drawn at $80 \%$ of maximum velocity, and, for both the skilled and the unskilled subject, the line intersects the mechanical efficiency curve at close to maximum. At present, the Asami et al. study is the only demonstration of a relationship between movement economy, accuracy of outcome, and level of skill.

Although these observations show greater economy in more experienced individuals, only relatively recently has experimental work been undertaken to systematically examine changes in movement coordination and control parameters with practice. In a study by Durand, Geoffroi, Varray, and Prefault (1994), changes in metabolic energy expenditure and movement parameters (amplitude and frequency) were examined as a function of practice on a ski-simulator. Metabolic cost increased significantly over sessions because the subjects were unconstrained with regard to movement amplitude and frequency, and they tended to perform more work as they became better practiced. When, however, movement cost was calculated, by dividing metabolic cost by the product of amplitude and frequency, the economy of performance, as measured by this variable, decreased over sessions.

Although, in the performance of everyday motor skills, work rate varies, as in Durand et al.'s (1994) study, in experiments undertaken by the present authors, external work rate was held constant in order to explore changes in coordination and control and movement economy by manipulating one category of constraint while the others remain fixed. For example, our data, shown in Figure 5, reveal the effect of manipulating the environment constraints of treadmill grade. In our earlier discussion of critical points, it was suggested that, with an increase in positive treadmill grade, the oxygen consumption curves would have crossed at the point at which an individual would make the transition from creeping to walking. In manipulating treadmill grade in this experiment, environment constraints were changed, while holding task and organism constraints constant. Thus, the sources of constraint identified in the framework (Figure 1) can be independently manipulated, and the manner in which the movement pattern is economically adapted to these changes observed.

Sparrow and Irizarry-Lopez (1987) and Sparrow and Newell (1994) also investigated changes in movement economy that were associated with practice at walking on hands and feet (creeping) on a motor-driven treadmill. 


\section{SUBJECT G}

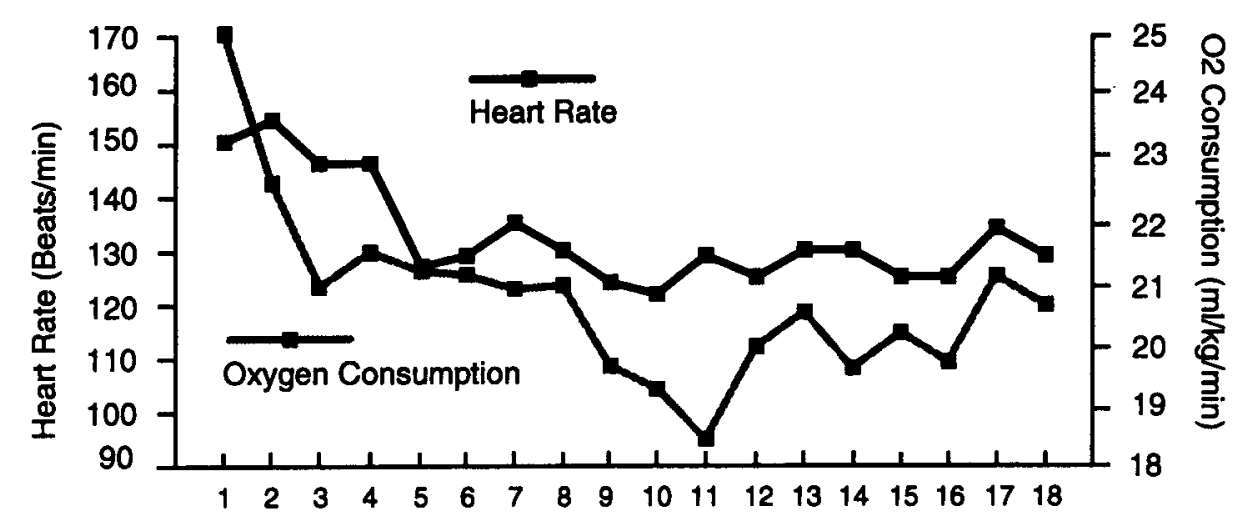

\section{SUBJECT M}

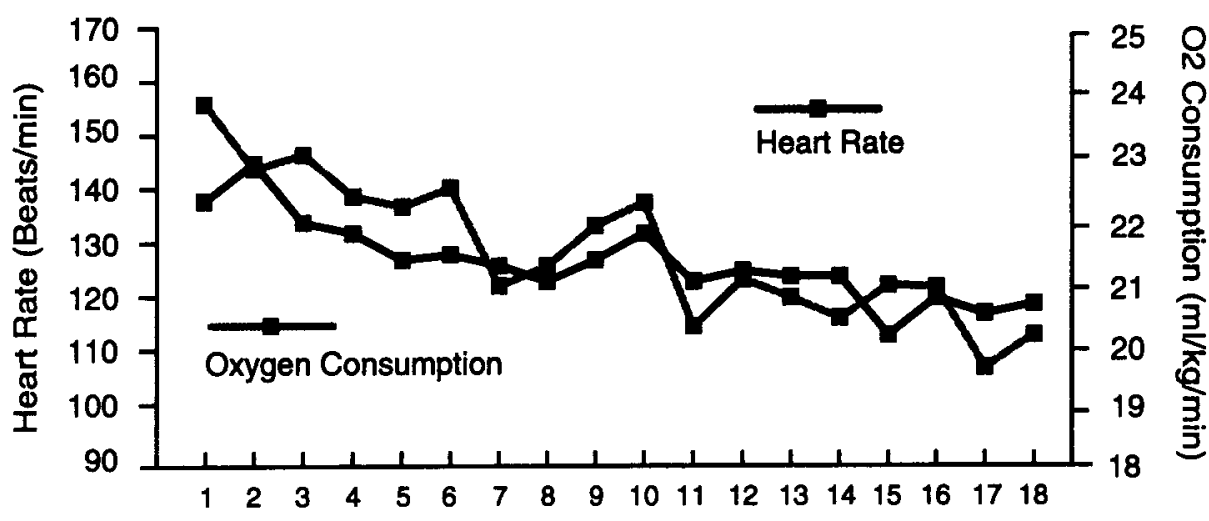

Figure 9. Metabolic and gait variables for 2 adult males creeping at an average speed of $2.3 \mathrm{kph}$ (1.4 mph) on a motor-driven treadmill at zero grade. There were eighteen 3-min learning trials (2 trials/session). (From "Energy Expenditure and Motor Performance Relationships in Humans Learning a Motor Task," by W. A. Sparrow and K. M. Newell, 1994, Psychophysiology, 31, p. 341. Copyright 1994 by Cambridge University Press. Reprinted with permission.)

In both studies, over days of practice, there was a statistically significant decrease in the metabolic cost of transporting the body a set distance. Practice-related changes in oxygen consumption and heart rate for 2 subjects in Sparrow and Newell's study are shown in Figure 9. The data show concomitant changes in oxygen consumption and heart rate over practice sessions, and both subjects showed a fairly systematic decrease in both variables over trials. Subject $\mathrm{G}$ showed little heart rate change after session three (trial 6), whereas his oxygen consumption continued to decrease up to session six (trial 11). The oxygen consumption data indicate, therefore, an overall improvement in economy with practice for both subjects.

The above findings raise the question as to how practice leads to an improvement in economy. Sparrow and Irizarry-Lopez's (1987) experiment clearly identified changes in both interlimb timing and intralimb coordi- nation, as measured by thigh-shank relative motion plots, that were correlated with metabolic cost. Thus, changes in the coordination and control characteristics of the creeping gait pattern were closely tied to changes in metabolic energy expenditure. These findings provide the strongest empirical support to date of the relationship between motor learning, economy, and related changes in the coordination and control of movement.

\section{Summary}

The observations in this section can be summarized by proposing three fundamental principles underlying the study of movement economy. The first derives from Hull (1943), to the effect that, when the organism is repeatedly presented with a number of equally reinforced alternative responses, that response requiring the least metabolic energy expenditure will become the dominant response. 
The second principle is that, when either the choice of response has been made or no choice given and a goal response specified, over trials the organism minimizes the metabolic energy expended in achieving the task goal. This economy-learning, or efficiency-learning, relationship, as shown in Figure 7, was first suggested by Freeman (1948), but Brener and Mitchell (1989) have also recognized the same principle. The third principle serves to explain these phenomena - the principle of least metabolic energy expenditure. In being receptive to sensory information about metabolic energy expenditure, organisms select the least effortful coordination and control function, and, with practice, the selected control parameters are refined to attain the task goal with less metabolic energy expenditure. The following section examines the contribution of various sources of informational support for response selection and metabolic energy minimization.

\section{INFORMATIONAL SUPPORT FOR REGULATING METABOLIC ENERGY EXPENDITURE}

The previous sections have presented evidence to show that the coordination and control of movement emerges as an economical response to task/environment constraints. This section describes how sensory information from receptor organs, resulting from any posture or movement, is utilized to regulate movement economy. Before discussing the informational support for metabolic energy regulation in detail, a brief historical overview of theorizing about sensory information and movement will be presented. This overview is important in showing why it has taken a long time for consideration of the sensory information mediating metabolic energy expenditure to emerge in the motor behavior literature.

\section{Historical Overview}

Woodworth's (1899) classic experiments on the accuracy of aiming movements highlighted the importance of visual information for movement control under conditions in which the response was sufficiently slow to allow visual information to be utilized. At about the same time, Sherrington (1947) coined the terminology for three classes of receptors conveying sensory information to the central nervous system. The triad comprised proprioceptors that monitor the state of the muscles, tendons, and joints; exteroceptors, giving information about the external environment, such as that obtained from vision; and interoceptors that signal the condition of the internal state of the organism with respect to temperature, blood pressure, digestive functions, and so on. It is important to note, however, that the term interoceptors, as coined by Sherrington, included specifically receptors of the digestive tract and their responses to chemical irritants, and this was the only meaning that Sherrington attached to the term interoception. By the 1930 s, receptors sensitive to chemical stimuli, labeled chemoreceptors had been discovered, and receptors sensitive to changes in blood pressure had been identified (baroreceptors) which also, as Chernigovskiy (1967) put it, did not find a place in Sherrington's definition. According to Chernigovskiy, the term interoceptors is therefore now taken to mean all the receptor organs of the digestive tract, the cardiovascular and lymphatic systems, and the internal organs.

Early work on maze learning by rats concerned the relative importance of these various forms of sensory information as conditioned stimuli. Following Watson's (1907) pioneering experiments, there was much research effort up to the late 1930s (see, e.g., Honzik, 1936; Hunter, 1930) that produced findings that challenged Watson's proposition that proprioceptive feedback was the necessary stimulus for maze learning. Lashley's deafferentation experiments (see, e.g., Lashley \& Ball, 1929; Lashley \& McCarthy, 1926), spurred perhaps by careful observations of both highly skilled performances and pathological motor responses, provided data that called into question the role of peripheral feedback and provoked theorizing about centrally stored sequences of muscle commands.

These pioneering studies of maze learning by rats and the attempts to determine the nature of the necessary stimuli for maze learning had a profound influence on the later theorizing of psychologists interested in human motor learning. Adams $(1968,1971)$ and Keele (1968) have perhaps been the most influential contemporary theorists on the issue of feedback and motor skill learning. Adams's contribution was to suggest that sensory feedback from movements can be used to guide learning by comparing the sensory feedback from the just-completed movement with the perceptual trace, which provided the reference mechanism in his closed-loop theory of motor learning. The theory incorporated the notion of sensory feedback as stimuli that are conditioned to learned responses, with the closed-loop error-centerd principles of control borrowed from engineering and biology. In the 1971 theory, it was made clear that the sensory feedback is not only proprioceptive stimuli, the obvious concomitant of movement, but also includes exteroceptive sources, such as visual, tactual, and auditory information. In a later review, Adams (1977) addressed the same controversy concerning the nature and contribution of sensory feedback in movement regulation - specifically, timing and positioning. Here, once more, it was proposed that, "There is no a priori reason why feedback from any sensory system cannot inform about movement" (p. 518).

Whereas Adams $(1968,1971)$ championed the view that sensory feedback from exteroceptive and proprioceptive sources is important for motor learning, Keele's (1968) notion of a motor program restimulated a behavioral consideration of open-loop mechanisms of motor control. Keele's position in the 1968 paper was that, under some circumstances, such as with very fast or well-learned movements, peripheral feedback does not influence the accuracy of movement production. This perspective was supported both by experiments with humans making rapid responses and by observations of functional motor patterns in surgically deafferented animals. This avenue 
of theorizing led to much research on motor programs, with efforts being made to discover the parameters of the movements that might be stored centrally in the form of a motor program (see, e.g., Shapiro, 1977; Shapiro, Zernicke, Gregor, \& Diestel, 1981; Summers, 1977). Keele's (1981) later definition of a motor program had a more important role for feedback, and today the centralperipheral discussion is less polarized than it was in the 1960 s and 1970s. Keele (1968) also provided the major theoretical impetus for studies concerned with the minimum amount of time required for visual information to be profitably used in aiming movements (see, e.g., Carlton, 1979; Keele \& Posner, 1968, Zelaznik, Hawkins, \& Kisselburgh, 1983).

In recent years, Lee (1978) has proposed the term exproprioceptive, to describe sensory information about body position in relation to the environment. This is usually visual information that serves not only to control responses but also to prepare the motor system for various contingencies in activities such as driving or locomotion. Studies of exproprioception have been mainly concerned with vision, with recent discussion of this phenomenon (see, e.g., Lee, 1978, 1980; Turvey, 1977) owing its greatest debt to Gibson's $(1950,1958)$ theorizing about the visual control of movement. The essence of this position is that the changing pattern of light at the eye, the optic flow field, specifies directly the spatial and temporal relationships between the observer and surrounding objects. For example, vision has been shown to work exproprioceptively (Lee, 1980) in controlling the time to contact between both stationary and moving objects and the individual's body or limbs.

This, in brief, is the legacy of theorizing about the function of sensory information in the learning and control of movement. Although never explicitly denied a role in motor behavior, sensory information about metabolic energy expenditure has not previously been considered important for regulating movement. One possible reason for this is that influential theorists, such as Adams and Keele, based their work on early learning experiments concerned with proprioceptive and visual feedback. In the following section, we consider the role of various forms of sensory information that might be utilized in the regulation of metabolic energy expenditure.

\section{Sensory Regulation of \\ Metabolic Energy Expenditure}

The most important sensory information regulating movement economy is that from interoceptors. Pioneering work on interoceptive conditioning was undertaken by Russian psychophysiologists in the 1940 s and $1950 \mathrm{~s}$, and Razran (1961) has provided a comprehensive and detailed review of this work. In these experiments, stimuli were delivered to human subjects primarily by inflating (with air or water) preexisting fistulas, implanted as a part of medical treatment, or gastric tubes and balloons swallowed for experimental purposes. A variety of similar and often more invasive techniques were used with nonhuman subjects (usually dogs). The fundamental observation from this literature is that sensory information produced by the stimulation of internal organs, such as the stomach and the bladder, can be conditioned to external or exteroceptive stimuli. In one experiment reported by Razran, for example, the urinary bladder fistulas of volunteer patients were inflated while the subjects were able to view dials with scale readings indicating the volume of air injected to distend the fistula. When the dials were later disconnected and sham readings presented, the subjects' measured bladder control and reported urinary sensations were consistent with the dial readings rather than with the actual urinary inflow. Thus, interoceptive stimuli can be conditioned to exteroceptive information, and, as we suggest later, it is reasonable to assume that sensory sensations associated with the metabolic response to exercise can also be conditioned to exteroceptive stimuli. For example, visual information about movements or features of the environment that afford least-energydemanding alternatives can, presumably, be conditioned to interoceptive stimuli concerning heart rate, body temperature, respiration, and other cardiovascular responses to exercise.

More recent evidence that supports the role of interoceptive feedback in controlling movement economy comes from experiments using biofeedback of internal states, in a manner similar to that of the early Russian experiment that Razran (1961) described. In contrast to the selfoptimization studies reviewed earlier, in biofeedback experiments, augmented sensory information, or augmented feedback, is provided by the experimenter. It has been shown that humans can learn to utilize this information to modify voluntary skeletal muscle activity during exercise. While exercising on a treadmill or bicycle ergometer, healthy subjects given cardiovascular augmented feedback have shown greater reductions in heart rate, systolic blood pressure, and pulse transit time than have no-feedback controls (see, e.g., Goldstein, Ross, \& Brady, 1977; Lo \& Johnston, 1984a, 1984b; Perski \& Engel, 1980). The exact nature of the biological parameters that the subject learns to control in these studies is uncertain; the effects are suggested to be due either to the control of voluntary muscle activity or to the influence of internal mechanisms, such as sympathetic control of the heart.

In addition to a concern with interoceptive stimuli as a source of sensory information in regulating movement economy, it is interesting to note that there has also been attention focused on the role of exteroceptive information in providing energy expenditure information. Most of the work on exteroceptive information has involved the role of vision in the control and learning of motor skills. As pointed out above, Warren (1984) showed that, in stair climbing, there is an optimal riser height proportional to the individual's leg length that minimizes metabolic energy expenditure. He also showed that individuals are capable of visually perceiving the optimal riser height. Judgments from photographic slides of preferred riser 
heights were consistent with the optimal riser heights derived from oxygen consumption measures. This suggests a visual perceptual basis to the regulation of economy, in which vision works to inform, in a prospective manner, about the likely energy expenditure of a task on the basis of body-scaled information. In other words, it appears that individuals can make a priori estimates of the metabolic energy expenditure of a specific movement pattern or movement sequence.

A further possibility is that, in some cases, visual exteroceptive information might be useful, primarily, for initially guiding the search for the most adaptive pattern of coordination and control, which, thereafter, is fine-tuned by interoceptive information in order to produce an economical control parameterization. From this point of view, energy minimization would not be given a leading role early in learning but would become more important later as the coordination pattern is refined or smoothed. Thus, as with the other sensory modalities, it might be useful to position metabolic energy minimization within the context of stages of learning, with sensitivity to metabolic cost playing a primary role in the later stages of learning, in the autonomous (Fitts \& Posner, 1967) or motor stages (Adams, 1971).

\section{Summary}

This section has provided an historical review of research related to the sensory information utilized in the learning and control of movement. Sensory information from internal organs, interoceptive information, has rarely been denied a role by students of motor behavior; rather, it has seldom been proposed as important for the acquisition and control of motor responses. Research by physiologists interested in the entrainment of motor and cardiovascular responses has implied that cardiovascular responses to exercise and timing characteristics of limb movements are causally related. It has been suggested, furthermore, that this relationship might be governed by the expedient of performing economically. Both human and nonhuman subjects appear to have the capacity to regulate the frequency, relative timing, and other aspects of the movement, in order to minimize energy expenditure.

\section{DIRECTIONS FOR FUTURE RESEARCH}

Movement economy is emerging as an important construct in contemporary theorizing about the coordination and control of movement. In this paper, we have identified three major subdomains of movement economy research: self-optimization, entrainment, and learning. In addition, we have proposed that, in all three of these subdomains, the observed pattern of movement coordination and control can be viewed as emerging from an interaction of constraints, with metabolic energy expenditure being the currency of the interaction. This review has provided a stimulus to research that is designed to test predictions concerning economy-related phenomena in the motor domain. In this section, future research directions in self-optimization, entrainment, and learning are identified. In addition, research questions are outlined concerning the informational support for movement economy, an issue that underlies research in the three subdomains.

\section{Self-Optimization}

In the section on self-optimization, reference was made to a comfort mode, or freely chosen rate, of performing repetitive motor tasks. In the mainstream of motor behavior research, only recently has interest been shown in the relationship between comfort, metabolic energy expenditure, and the emergent movement pattern (see, e.g., Holt et al., 1995). It would be interesting to investigate this phenomenon more closely. For example, when in one experiment we (Sparrow \& Newell, 1994) operationalized comfort by requesting subjects to select a preferred treadmill grade, only 1 of 3 subjects selected a more economical grade than that imposed by the experimenters. This finding suggests that, in some motor tasks, subjective feelings of comfort or exertion might be the primary stimulus in organizing a preferred mode, rather than metabolic energy expenditure. In most cases, however, it would be expected that the metabolic response to exercise is strongly correlated with comfort or exertion, as is shown in an extensive literature based on the perceived exertion research of Borg (e.g., Borg, 1973). An interesting avenue of research would be to examine various operational definitions of comfort mode and their relation to metabolic energy expenditure and the emergent movement pattern.

In addition to the question of how preferred modes are operationalized, there is also the question of how preferred modes are established. Preferred modes may be dependent on the physiological and anthropometric characteristics of the organism that act as constraints on the movement pattern. Such characteristics are those related to limb length, limb segment lengths and proportions, segmental mass, location of center of moment, joint range of motion, muscle fiber type, muscle mass, and the capacity of the muscle-joint complex to produce force. In recent work on stepping over obstacles, for example, Sparrow, Shinkfield, Chow, and Begg (1996) hypothesized that, in locomoting through the everyday environment, lower limb trajectories are modulated in order to clear obstacles safely while conserving metabolic energy. To test the energy conservation hypothesis, it would be necessary to measure the metabolic energy expended in negotiating obstacles in order to determine whether the subjectselected strategies for going over and around obstacles demand less metabolic energy than those imposed by the experimenter. To date this has not been done. Nevertheless, there is considerable potential for investigating locomotor strategies in terms of self-optimization given organism constraints, such as leg length, and task constraints, such as the obstacle's height and rigidity.

A further potential avenue of inquiry is the relationship between metabolic energy expenditure and abrupt tran- 
sitions in coordination. Such abrupt transitions, or sudden jumps (Turvey, 1990), have been of considerable interest to contemporary motor behavior theorists. When sudden jumps in coordination are observed, there is a fundamental reorganization of the coordination mode, and, as is shown by Hoyt and Taylor's (1981) data in Figure 4, such jumps are associated with minimum metabolic energy expenditure. Although many examples of transitions in interlimb coordination have been documented for human subjects, there have been few attempts to link transitions in coordination directly to metabolic energy expenditure. At present, the authors are examining the link between transitions in coordination and changes in metabolic energy expenditure, in order to further test the hypothesis that critical thresholds of metabolic energy expenditure are the stimulus for abrupt reorganization of interlimb coordination.

\section{Entrainment}

Systematic research on entrainment is very recent. Kirby et al. (1989), for example, were the first to demonstrate coupling between the cardiac cycle and a movement cycle. There is, therefore, a range of entrainment phenomena that are, as yet, unexplored. The fundamental research question is whether, across a range of movement skills and for all individuals, there are characteristic stable phase relationships between metabolic variables and movement parameters. If future research were to confirm these observations, the question would arise as to the biological significance of such phase coupling. Earlier, it was proposed that the stable phase relationships described as entrainment may be associated with metabolic energy conservation in that a close time-locking of the respiratory and cardiac cycle with critical events in the movement cycle, such as the point of maximum force production, may produce a more stable and economical performance than one in which metabolic and movement cycle variables are out of phase.

An interesting observation made by Bramble and Carrier (1983) is that entrainment may be learned. If entrainment is related to economy, learning to entrain the respiratory or cardiac cycle with the movement cycle may be a mechanism by which greater economy of metabolic energy expenditure is achieved. Thus, experiments designed to determine the effect of practice on entrainment would make an important contribution to understanding long-term adaptations to exercise. Such experiments would be particularly interesting if entrainment could be shown to covary with metabolic energy expenditure measured with expired air techniques. Further suggestions for examining the learning/economy relationship are made below.

\section{Learning}

With respect to learning new movement sequences, it would be valuable to investigate further the process by which movements are initially selected and later refined or fine-tuned with practice in order to minimize metabolic cost. With the framework outlined here, the expedient to move economically can be usefully viewed as guiding the search for the optimal coordination and control solutions to the problem of overcoming task/environment constraints. To date, there have been very few demonstrations of the relationship between metabolic energy expenditure and practice in which either discrete (or qualitative) transitions in coordination or continuous change in control parameters were linked directly to changes in metabolic variables, such as oxygen consumption, respiration, and heart rate. Our observation is that, in repetitive gross-motor activities, such as creeping and ergometer rowing, movements tend to increase in amplitude and decrease in frequency with practice. We suggest, therefore, that a longer-slower hypothesis will be supported in studies of practice-related adaptations of movements to fixed task constraints. The hypothesis that changes in timing are linked to reductions in oxygen consumption is consistent with the long-standing observation of an optimum speed of muscle contraction that maximizes mechanical work relative to chemical energy degraded (see, e.g., Hill, 1922). Considerably more work remains to be done, however, to fully elaborate the relationship between metabolic expenditure and changes in control parameters, such as amplitude and timing.

In characterizing motor learning as a search for an economical movement pattern, a general research strategy, based on the framework presented here, would be to investigate the interaction of task, environment, and organism constraints by systematically manipulating one source of constraint while holding the others constant. In our work, we have tended to hold the task constraints fixed, as in creeping on a treadmill at a constant speed, while manipulating the environment constraint of treadmill grade. In future work, we plan to examine the influence of organism constraints either by comparing the movement patterns of groups of subjects with different anthropometric characteristics or by experimentally restricting movement at one or more joints. The latter strategy has been employed by Inman, Ralston, and Todd (1981) in studies of the energetics of human walking.

\section{Informational Support for Movement Economy}

A critical question arising from the discussions of self-optimization, entrainment, and learning is that concerning the process by which movements are modified to be economical in the absence of augmented information about the optimal coordination and control parameters. In the review of learning, it was hypothesized that metabolic energy expenditure is the basis for response selection and refinement, and it was shown that traditional feedback-oriented theorizing about motor skill acquisition has given little consideration to mechanisms regulating metabolic energy expenditure. In contemporary theorizing about action, however, the manner in which organisms conserve and expend metabolic energy is an important theme (see, e.g., Kelso, 1990; Kugler \& Shaw, 1990). Haken (1991), for example, has speculated that 
metabolic energy expenditure could be a macroscopic variable governing the neural networks mediating motor responses. We propose, therefore, that future research could profitably examine metabolic energy expenditure directly, using expired air methods, in order to further our understanding of the link between metabolic energy expenditure and the emergent movement pattern.

Although it is not possible to investigate the role of interoceptive sensory information by monitoring performance when it is eliminated, experimental procedures can be devised in which interoceptive information is augmented. For example, experiments are presently being conducted by the authors that examine the effects of augmented oxygen consumption information by comparing performance economy in a practice phase with oxygen consumption feedback with a baseline phase in which oxygen consumption feedback is withdrawn. This is promising to be an interesting avenue of research from a theoretical perspective in explaining how movements are refined with practice. In addition, the application of these findings to improving performance in a variety of motor skills is an important direction for future work. Preliminary findings suggest that providing augmented information about the metabolic responses to exercise can assist the performer in modifying movements so as to become more economical in achieving the task goal.

\section{CONCLUDING REMARKS}

In conclusion, it is important to consider the broader significance of research into movement economy. In all human movement domains, there are benefits associated with developing the means to explain, predict, and control human behavior in order to achieve greater economy. In the home, in the workplace, and in sport, greater economy of movement allows either more to be done in a given time or the same task to be completed with metabolic energy to spare. In all human motor skills, there is, therefore, an advantage to be gained by those who are most economical in their movements. For all animals in their natural environment, survival advantages are conferred on those individuals able to perform life-sustaining behaviors with the least metabolic energy consumption. Our conclusion from the literature reviewed here is that the performance of everyday motor skills in the manmade environment may be constrained by the same imperative.

\section{REFERENCES}

Adams, J. A. (1968). Response feedback and learning. Psychological Bulletin, 70, 486-504.

ADAMS, J. A. (1971). A closed-loop theory of motor learning. Journal of Motor Behavior, 3, 111-149.

ADAMS, J. A. (1977). Feedback theory of how joint receptors regulate the timing and positioning of a limb. Psychological Review, 84, 504-523.

AleSHINSKY, S. Y. (1986). An energy "sources" and "fractions" approach to the mechanical energy expenditure problem: Il. Movement of the multi-link model. Journal of Biomechanics, 19, 295-300.
ANDERSON, T. (1996). Biomechanics and running economy. Sports Medicine, 22, 76-89.

asami, T., Togari, H., Kikuchi, T., Adachi, N., Yamamoto, K., Kita GAWA, K., \& SANO, Y. (1976). Energy efficiency of ball kicking. In P. V. Komi (Ed.), Biomechanics (Vol. 13, pp. 135-139). Baltimore: University Park Press.

Bannister, R. G., Cunningham, D. J. C., \& Douglas, C. G. (1954), The carbon dioxide stimulus to breathing in severe exercise. Journal of Physiology, 125, 90-117.

Bechbache, R. R., ChOW, H. H. K., Duffin, J., \& ORsini, E. C. (1979). The effects of hypercapnia, hypoxia, exercise and anxiety on the pattern of breathing in man. Journal of Physiology, 293, 285-300.

BECHBACHE, R. R., \& DufFin, J. (1977). The entrainment of breathing frequency by exercise rhythm. Journal of Physiology, 272, 553-561.

Beek, P. J., PePer, C. E., \& van Wieringen, P. C. W. (1992). Frequency locking, frequency modulation, and bifurcations in dynamic movement systems. In G. E. Stelmach \& J. Requin (Eds.), Tutorials in motor behavior II (pp. 599- 622) Amsterdam: Elsevier.

BERNSTEIN, N. A. (1967). The coordination and regulation of movements. London: Pergamon.

BOBBERT, A. C. (1960). Energy expenditure in level and grade walking. Journal of Applied Physiology, 15, 1015-1021.

BoRG, G. A. V. (1973). Perceived exertion: A note on history and methods. Medicine \& Science in Sport, 5, 90-93.

Bramble, D. M., \& CarRIER, D. R. (1983). Running and breathing in mammals. Science, 219, 251-256.

Brancazio, P. J. (1981). Physics of basketball. American Journal of Physics, 49, 356-365.

BRENER, J. (1986). Operant reinforcement, feedback and the efficiency of learned motor control. In G. H. Coles, E. Donchin, \& S. W. Porges (Eds.), Psychophysiology: Systems, processes and applications (pp. 309-327). New York: Guilford.

BRENER, J. (1987). Behavioral energetics: Some effects of uncertainty on the mobilization and distribution of energy. Psychophysiology, 24, 499-512.

BRENER, J., \& MitCheLl, S. (1989). Changes in energy expenditure and work during response acquisition in rats. Journal of Experimental Psychology: Animal Behavior Processes, 15, 166-175.

BRENER, J., PHILliPS, K., \& SHERWOOD, A. (1983). Energy expenditure during response-dependent and response-independent food delivery in rats. Psychophysiology, 20, 384-392.

Carlton, L. G. (1979). Control processes in the production of discrete aiming responses. Journal of Human Movement Studies, 5, 115-124.

Cavanagh, P. R., \& Kram, R. (1985a). The efficiency of human movement-a statement of the problem. Medicine \& Science in Sports \& Exercise, 17, 304-308.

CavanaGh, P. R., \& KRam, R. (1985b). Mechanical and muscular factors affecting the efficiency of human movement. Medicine \& Science in Sports \& Exercise, 17, 326-331.

Cavanagh, P. R., \& Williams, K. R. (1982). The effect of stride length variation on oxygen uptake during distance running. Medicine \& Science in Sports \& Exercise, 14, 30-35.

ChernigovskiY, V. N. (1967). Interoceptors. Washington, DC: American Psychological Association.

CHOW, C. J., \& JACOBSON, D. H. (1971). Studies of human locomotion via optimal programming. Mathematical Biosciences, 10, 239-306.

Coleman, W. M. (1921). The psychological significance of bodily rhythms. Journal of Comparative \& Physiological Psychology, 1, 213-220

DE CAMP, J. E. (1920). Relative distance as a factor in the white rat's selection of a path. Psychobiology, 2, 245-253.

DeVRIES, H. A., \& Housh, T. J. (1994). Physiology of exercise for physical education, athletics and exercise science (5th ed.). Dubuque, IA: Brown \& Benchmark.

Diedrich, F. J., \& WARREN, W.H., JR. (1995). Why change gaits? Dynamics of the walk-run transition. Journal of Experimental Psychology: Human Perception \& Performance, 21, 183-202.

Durand, M., Geoffroi, V., Varray, A., \& Prefault, C. (1994). Study of the energy correlates in the learning of a complex self-paced cyclical skill. Human Movement Science, 13, 785-799. 
FALLS, H. B., \& HUMPHREY, L. D. (1976). Energy cost of walking and running in young women. Medicine \& Science in Sports, 8, 9-13.

FitTs, P. M., \& Posner, M. I. (1967). Human performance. Belmont, CA: Brooks/Cole.

FLASH, T. (1990). The organization of human arm trajectory control In J. M. Winters \& S. L-Y. Woo (Eds.), Multiple muscle systems. Biomechanics and movement organization (pp. 283-301). New York: Springer-Verlag.

FREEMAN, G. L. (1948). The energetics of human behavior. Ithaca, NY: Cornell University Press.

GiBson, J. J. (1950). The perception of the visual world. Boston: Houghton Mifflin

Gibson, J. J. (1958). Visually controlled locomotion and visual orientation in animals. British Journal of Psychology, 49, 182-194.

Goldstein, D. S., Ross, R. S., \& BradY, J. V. (1977). Biofeedback heart rate training during exercise. Biofeedback \& Self Regulation, 2 , 107-126.

GuTHRIE, E. R. (1935). The psychology of learning. New York: Harper.

HAKEN, H. (1991). Synergetics of movement coordination: Reaction to Bullock and Grossberg. Human Movement Science, 10, 113-115.

HaKen, H, Kelso, J. A. S., \& BUnz, H. (1985). A theoretical model of phase transitions in human hand movements. Biological Cybernetics, $51,347-356$

HiLl, A. V. (1922). The maximum work and mechanical efficiency of human muscles and their most economical speed. Journal of Physiology, 56, 19-41.

HogAN, N., \& FLASH, T. (1987). Moving gracefully: Quantitative theories of motor coordination. Trends in Neurosciences, 10, 170-174.

Holt, K. G., Jeng, S. F., Ratcliffe, R., \& Hamill, J. (1995). Energetic cost and stability during human walking at the preferred stride frequency. Journal of Motor Behavior, 27, 164-178.

HonzIK, C. H. (1936). The sensory basis of maze learning in rats. Comparative Psychology Monographs, 13 (Whole No.64).

HoYT, D. F., \& TAYLOR, C. R. (1981). Gait and the energetics of locomotion in horses. Nature, 292, 239-240.

HreluaC, A. (1993). Preferred and energetically optimal gait transition speeds in human locomotion. Medicine \& Science in Sports \& Exercise, 25, 1158-1162.

HuLl, C. L. (1943). Principles of behavior. New York: Appleton-Century

HUNTER, W. S. (1930). A further consideration of the sensory control of the maze habit in the white rat. Journal of Genetic Psychology, 38, 3-19.

InMAN, V. T., Ralston, H. J., \& Todd, F. (1981). Human walking. Baltimore: Wilkins.

Kay, J. D. S., Petersen, E. S., \& Vejby-Christensen, H. (1975) Breathing in man during steady-state exercise on the bicycle at two pedalling frequencies, and during treadmill walking. Journal of Physiology, 251, 645-656.

KeELE, S. W. (1968). Movement control in skilled motor performance. Psychological Bulletin, 70, 387-403.

KEELE, S. W. (1981). Behavioral analysis of motor control. In V. B Brooks (Ed.), Handbook of physiology: Section 1. Vol. 2: Motor con trol (pp. 1391-1413). Bethesda, MD: American Physiological Society.

Keele, S. W., \& Posner, M. I. (1968). Processing of visual feedback in rapid movements. Journal of Experimental Psychology, 77, 155-158.

Kelso, J. A. S. (1990). Phase transitions: Foundations of behavior. In H. Haken \& M. Stadler (Eds.), Synergetics of cognition (pp. 249-268). Heidelberg: Springer-Verlag.

Kirby, R. L., Nugent, S. T., Marlow, R. W., Macleod, D. A., \& MarBLE, A. E. (1989). Coupling of cardiac and locomotor rhythms. Journal of Applied Physiology, 66, 323-329.

KREBS, J. R. (1978). Optimal foraging: Decision rules for predators. In J. R. Krebs \& N. B. Davies (Eds.), Behavioral ecology: An evolutionary approach (pp. 23-63). Oxford: Blackwell.

Kugler, P. N., Kelso, J. A. S., \& Turvey, M. T. (1980). On the concept of coordinative structures as dissipative structures: 1. Theoretical lines of convergence. In G. E. Stelmach \& J. Requin (Eds.), Tutorials in motor behavior (pp. 3-47). Amsterdam: North-Holland.

Kugler, P. N., Kelso, J. A.S. , \& Turvey, M. T. (1982). On the control and coordination of naturally developing systems. In J. A. S. Kelso \&
J. E. Clark (Eds.), The development of movement control and coordination (pp. 5-78). New York: Wiley.

KUGLER, P. N., \& SHAW, R. E. (1990). Symmetry and symmetry-breaking in thermodynamic and epistemic engines: A coupling of first and second laws. In H. Haker \& M. Stadler (Eds.), Synergetics of cognition (pp. 296-331). Heidelberg: Springer-Verlag.

KUGLER, P. N., \& TURVEY, M. T. (1987). Information, natural law, and the self-assembly of rhythmic movement. Hillsdale, NJ: Erlbaum.

Kuo, Z. Y. (1922). The nature of unsuccessful acts and their order of elimination in animal learning. Journal of Comparative Psychology, 2, 1-27.

LASHLEY, K. S., \& BALL, J. (1929). Spinal conduction and kinaesthetic sensitivity in the maze habit. Journal of Comparative Psychology, 9, 71-106.

LASHLEY, K. S., \& MCCARTHY, D. A. (1926). The survival of the maze habit after cerebellar injuries. Journal of Comparative Psychology, 6, 423-434.

LEE, D. N. (1978). The function of vision. In H. Pick \& E. Salzmann (Eds.), Modes of perceiving and processing information (pp. 159-170). Hillsdale, NJ: Erlbaum.

LEE, D. N. (1980). Visuo-motor coordination in space time. In G. E. Stelmach \& J. Requin (Eds.), Tutorials in motor behavior (pp. 281-295). Amsterdam: North-Holland.

LIN, D. C. (1980). Optimal movement patterns of the lower extremity in running. Unpublished doctoral dissertation, University of Illinois at Urbana-Champaign.

Lo, C. R., \& Johnston, D. W. (1984a). Cardiovascular feedback control during dynamic exercise. Psychophysiology, 21, 199-206.

Lo, C. R., \& Johnston, D. W. (1984b). The effect of the cardiovascular response to exercise using feedback of the product of interbeat interval and pulse transit time. Psychosomatic Medicine, 46, 115-125.

Mahler, D. A., Shuhart, C. R, Brew, E. \& Stukel, T. A. (1991). Ventilatory responses and entrainment of breathing during rowing. Medicine \& Science in Sports \& Exercise, 23, 186-192.

MARGARIA, R. (1976). Biomechanics and energetics of muscular exercise. Oxford: Oxford University Press, Clarendon Press.

Margaria, R., Cerretelli, P., Aghemo, P., \& Sassi, G. (1963). Energy cost of running. Journal of Applied Physiology, 18, 367-370.

MCCULLOCH, T. L. (1934). Performance preferential of the white rat in force-resisting and spatial dimensions. Journal of Comparative Psychology, 18, 85-111.

Nelson, W. L. (1983). Physical principles for economies of skilled movements. Biological Cybernetics, 46, 135-147.

NewELL, K. M. (1985). Coordination, control and skill. In D. Goodman, I. Franks, \& R. Wilberg (Eds.), Differing perspectives in motor control (pp. 295-317). Amsterdam: North-Holland.

NEWELL, K. M. (1986). Constraints on the development of coordination. In M. G. Wade \& H. T. A. Whiting (Eds.), Motor development in children: Aspects of coordination and control (pp. 341-360). Boston: Martinus Nijhoff

NORDEEN-SNYDER, K. (1977). The effect of bicycle seat height variation upon oxygen consumption and lower limb kinematics. Medicine \& Science in Sports, 9, 113-117.

NotTerman, J. M., \& MinTz, D. E. (1965). Dynamics of response. New York: Wiley.

Patterson, W. M. (1916). The rhythm of prose. New York: Columbia University Press

Perski, A., \& ENGEL, B. T. (1980). The role of behavioral conditioning in the cardiovascular response to exercise. Biofeedback \& Self Regulation, 5, 91-104.

Poole, P. M., \& Ross, B. (1983). The energy cost of sheep shearing. Search, 14, 3-4.

Poulton, E. C. (1957). On prediction in skilled movements. Psychological Bulletin, 54, 467-478.

RazRAN, G. (1961). The observable unconscious and the inferable conscious in current Soviet psychophysiology: Interoceptive conditioning, semantic conditioning, and the orienting reflex. Psychological Review, 68, 81-147.

RoBB, M. D. (1972). The dynamics of motor-skill acquisition. Englewood Cliffs, NJ: Prentice-Hall. 
Rosenbaum, D. A., Loukopoulos, L. D., Meulenbroek, R. G. J. Vaughan, J., \& ENGELBRecht, S. E. (1995). Planning reaches by evaluating stored postures. Psychological Review, 102, 28-67.

SALVENDY, G. (1972). Physiological and psychological aspects of paced and unpaced performance. Acta Physiologica Academiae Scientiarum Hungaricae, 42, 257-275.

Salvendy, G., \& Pilrtsis, J. (1974). Improvements in physiological performance as a function of practice. International Journal of Production Research, 12, 519-531.

Sams, C. F., \& Tolman, E. C. (1929). Time discrimination in white rats. Journal of Comparative Psychology, 5, 255-263.

SCнміDT, R. A. (1982). Motor control and learning: A behavioral emphasis. Champaign, IL: Human Kinetics.

SEASHORE, R. H. (1926). Studies in motor rhythm. Psychological Monographs, 36, 142-189.

ShaPIRO, D. C. (1977). A preliminary attempt to determine the duration of a motor program. In D. M. Landers \& R. W. Christina (Eds.), Psychology of motor behavior and sport (Vol. 1, pp. 17-24). Urbana, IL: Human Kinetics.

Shapiro, D. C., Zernicke, R. F., Gregor, R. J., \& Diestel, J. D. (1981). Evidence for generalized motor programs using gait pattern analysis. Journal of Motor Behavior, 13, 33-47.

SHERRINGTON, C. S. (1947). The integrative action of the nervous system. New Haven: Yale University Press. (Original work published 1906)

Sherwood, A., Allen, M. T., Obrist, P. A., \& Langer, A. W. (1986). Evaluation of beta-adrenergic influences in cardiovascular and metabolic adjustments to physical and psychological stress. Psychophysiology, 23, 89-104.

SINGER, R. N. (1968). Motor learning and human performance. London: Collier-Macmillan.

Solomon, R. L. (1948). The influence of work on behavior. Psychological Bulletin, 45, 1-40.

SPARROW, W. A. (1983). The efficiency of skilled performance. Journal of Motor Behavior, 15, 237-261.

SPARROW, W. A. (1992). Measuring changes in coordination and control. In J. J. Summers (Ed.), Approaches to the study of motor control and learning (pp. 147-162). Amsterdam: Elsevier.

SPARRow, W. A. \& Hughes, K. (1997, April). Minimum principles in human learning: The effects of practice and non-preferred work rates on metabolic energy expenditure and perceived exertion. Paper presented at the 24th Annual Experimental Psychology Conference, Deakin University, Victoria, Australia.

SParrow, W. A., \& IrIZarry-Lopez, V. M. (1987). Mechanical efficiency and metabolic cost as measures of learning a novel gross motor task. Journal of Motor Behavior, 19, 240-264.

SparRow W. A., \& Newell, K. M. (1994). Energy expenditure and motor performance relationships in humans learning a motor task. Psychophysiology, 31, 338-346.

Sparrow, W. A., Shinkfield, A. J., Chow, S., \& Begg, R. K. (1996) Characteristics of gait in stepping over obstacles. Human Movement Science, 15, 605-622.

Steinacker, J. M., Both, M., \& Whipp, B. J. (1993). Pulmonary mechanics and entrainment of respiration and stroke rate during rowing. International Journal of Sports Medicine, 14 (Suppl. 1), S15-S19.

SUmmers, J. J. (1977). The relationship between the sequencing and timing components of a skill. Journal of Motor Behavior, 9, 49-59.
Thompson, M. E. (1944). An experimental investigation of the gradient of reinforcement in maze learning. Journal of Experimental Psychology, 34, 390-403.

Tolman, E. C. (1932). Purposive behavior in animals and men. New York: Century,

TSAI, L. S. (1932). The laws of minimum effort and maximum satisfaction in animal behaviour. Monograph of the National Institute of Psychology [Peiping, China], No. 1. [Abstracted in Psychological Abstracts (1932), 6, No. 4329]

TURNER, J. R., \& CARROLL, D. (1985). Heart rate and oxygen consumption during mental arithmetic, a video game, and graded exercise: Further evidence of metabolically-exaggerated cardiac adjustments? Psychophysiology, 21, 261-267.

TURVEY, M. T. (1977). Preliminaries to a theory of action with reference to vision. In R. Shaw \& J. Bransford (Eds.), Perceiving, acting, and knowing (pp. 211-265). Hillsdale, NJ: Erlbaum.

TuRvey, M. T. (1990). Coordination. American Psychologist, 45, 938-953.

Turvey, M. T., \& FitzPatRick, P. (1993). Commentary: Development of perception-action systems and general principles of pattern formation. Child Development, 64, 1175-1190

Uno, Y., KaWATo, M., \& SuzUKI, R. (1989). Formation and control of optimal trajectory in human multijoint arm movement. Biological Cybernetics, 61, 89-101.

WANN, J. (1987). Trends in refinement and optimization of fine-motor trajectories: Observations from an analysis of the handwriting of primary school children. Journal of Motor Behavior, 19, 13-37.

WARREN, W. H. (1984). Perceiving affordances: Visual guidance of stair climbing. Journal of Experimental Psychology: Human Perception \& Performance, 10, 683-703.

WATERS, R. H. (1937). The principle of least effort in learning. Journal of General Psychology, 16, 3-20.

WATSON, J. B. (1907). Kinaesthetic and organic sensations: Their role in the reactions of the white rate to the maze. Psychological Review Monograph Supplements, 8 (2, Whole No. 33).

WHIPP, B. J., \& WASSERMAN, K. (1969). Efficiency of muscular work. Journal of Applied Physiology, 26, 644-648.

WINTER, D. A. (1979). A new definition of mechanical work done in human movement. Journal of Applied Physiology, 46, 79-83.

WOODWORTH, R. S. (1899). The accuracy of voluntary movement. Psychological Review, 3 (Suppl. 2, Whole No. 13).

Wyndham, C. H., Morrison, J. F, Williams, C. G., Strydom, N. B., von Rahden, M. J. E., Holdsworth, L. D., van Grann, C. H., \& VAN RENSBURG, A. J. (1966). Inter- and intra-individual differences in energy expenditure and mechanical efficiency. Ergonomics, 9, 17-29.

YoshioKA, J. G. (1929). Weber's law in the discrimination of maze distance by the white rat. University of California Publications in Psychology, 4, 155-184.

ZelazniK, H. N., HaWkins, B., \& Kisselburgh, L. (1983). Rapid visual feedback processing in single-aiming movements. Journal of Motor Behavior, 15, 217-236.

ZIPF, G. K. (1949). Human behavior and the principle of least effort. Cambridge, MA: Addison-Wesley.

(Manuscript received May 9, 1996 revision accepted for publication May 21, 1997.) 